Original Research Paper

\title{
Effects of Enriching Nitrogen and Phosphorus on the Growth of Sargassum Podacanthum Cultured in Potassium-Fortified Inland Saline Water
}

\author{
Ha Thi Thu Bui, Trong Quoc Luu and Ravi Fotedar \\ School of Molecular and Life Sciences, \\ Curtin University, Kent Street, Bentley, Perth, Western Australia, 6102, Australia
}

Article history

Received: 04-12-2017

Revised: 01-02-2018

Accepted: 05-04-2018

Corresponding Author:

Ha Thi Thu Bui

School of Molecular and Life

Sciences, Curtin University,

Kent Street, Perth, Western,

Australia, 6102, Australia

Email: ha.bui@student.curtin.edu.au

\section{Introduction}

Mariculture, including seaweed culture, in Inland Saline Water (ISW) is considered as a potential expansion and diversification of aquaculture industry in Australia (Allan et al., 2001). Seaweed culture can make use of salt-affected agricultural farms as it is less constrained by additional requirement for resources and changes in infrastructure than the culture of marine finfish and crustacean species. Therefore, growing Sargassum, in ISW can provide another source of

\begin{abstract}
Potassium-fortified inland saline water ( $\left.\mathrm{K}^{+} \mathrm{ISW}\right)$ has shown potential for growing marine species, including seaweed species. The response of a brown seaweed species, Sargassum podacanthum, to nitrogen and phosphorus enrichments were evaluated by culturing the species for 84 days in $\mathrm{K}^{+} \mathrm{ISW}$ and comparing it with Ocean Water (OW). The culture media were enriched weekly with ammonium chloride and sodium dihydrogen phosphate, with ammonium and phosphate ratios of 10:1 at five different concentrations $80: 8,120: 12,160: 16,200: 20$ and 240:24 $\mu \mathrm{M}$. The culture medium with no enrichment was used as a control. The water quality and biomass of $S$. podacanthum were measured fortnightly. The $S$. podacanthum biomass increase significantly with different concentrations of the nutrient supplementations. The standing biomass and Specific Growth Rate (SGR) of $S$. podacanthum were similar in $\mathrm{OW}$ and $\mathrm{K}^{+} \mathrm{ISW}$ in the absence of any nutrient supplementation and at the supplement concentration of ammonium and phosphate $160: 16 \mu \mathrm{M}$. However, from day 42 onwards, at the ratios of $80: 8,120: 12,200: 20$ and 240:24, S. podacanthum cultured in OW grew significantly faster than in $\mathrm{K}^{+} \mathrm{ISW}$. In $\mathrm{K}^{+} \mathrm{ISW}$, optimal growth of $S$. podacanthum was observed at the 160:16 and the increase in biomass was significantly higher than the initial biomass until day 70, whereas at the other four nutrient supplement concentrations, the $S$. podacanthum biomass remained unchanged during the entire culture period. The nitrite, total Kjeldahl nitrogen and phosphate concentrations in water were found to be significantly $(\mathrm{p}<0.05)$ and negatively correlated $(\mathrm{p}<0.05)$ with $S$. podacanthum biomass. Therefore, the results showed that the enrichment of $160 \mu \mathrm{M}$ ammonium and $16 \mu \mathrm{M}$ phosphate is required in the $\mathrm{K}^{+} \mathrm{ISW}$ for $S$. podacanthum to achieve optimal growth.
\end{abstract}

Keywords: Inland Saline Water, Sargassum podacanthum, Nutrient Enrichment, Potassium Fortification, Ammonium, Phosphate commodity to the farmers with a lower capital investment than farming in the sea (Borowitzka, 1997) and can be an additional tool to protect the inland environment in Australia by combating the salinity problems (Ogburn, 1997).

At the same salinity, the level of potassium $\left(\mathrm{K}^{+}\right)$ concentration in ISW is lower than in Ocean Water (OW) in Australia (Allan and Fielder, 1997; Dinh, 2016) and USA (Boyd and Thunjai, 2003; Forsberg et al., 1996) although other ionic profiles can be similar (Fotedar et al., 2011; Prangnell and Fotedar, 2006a). Potassium is vital 
for aquaculture and $\mathrm{K}^{+}$deficiency in ISW can negatively affect the growth of the aquatic animals (Mourad et al., 2012). For example, the survival of juvenile mulloway (Argyrosomus hololeptidotus) (Doroudi et al., 2006), juvenile snapper (Pagrus auratus) (Fielder et al., 2001) and red drum (Sciaenops ocellatus) (Forsberg et al., 1996) is adversely affected when cultured in low $\mathrm{K}^{+}$ environment. Therefore, fotifying ISW with $\mathrm{K}^{+}$to achieve similar concentration in $\mathrm{OW}$ is essential to sustain the growth of shrimp (Prangnell and Fotedar, 2006b; Tantulo and Fotedar, 2006), fish (Fielder and Allan, 2003), red seaweed Lomentaria sp. (Bui et al., 2017a) and brown seaweed Sargassum linearifolium (Bui et al., 2017b).

Sargassum spp. commonly used as a source of fertilizers and soil conditioners (Huisman, 2000), are the dominant taxa in near shore reef areas along Perth beaches (Womersley, 1996). Sargassum spp. are prevalent sources of compounds used in pharmaceutical (Hur et al., 2008) and agriculture industries (Ara et al., 1997). The extracts from Sargassum can be used as in the treatment of neurological disorders (Natarajan et al., 2009), dementia (Pangestuti and Kim, 2010) and HIV (Thuy et al., 2015). The fact that Sargassum is active in antioxidant activity, cholinesterase inhibition activity, neuroprotective activity, anti-cancer and cytotoxic activity has made it a popular ingredient in health enhancement products (Yende et al., 2014). S. fusiforme has been cultivated in Korea and Japan as a food source (Bast, 2014). Sargassum has many branches growing from a short stipe (Huisman, 2000). The length of Sargassum's thallus is about $0.1-2 \mathrm{~m}$, while its stipes are $1-20 \mathrm{~cm}$ long from a discoid-conical holdfast (Womersley, 1987). Of the Sargassum species, $S$. podacanthum is distributed from Point Peron (Western Australia (WA) to Port Noarlunga (South Australia) (Womersley, 1987) and thus can be an important candidate for growing in local ISW. The branches of $S$. podacanthum are typically terete, but more angular at the top, usually with short, scatter spines, which branch out radically (Womersley, 1987). S. podacanthum is thalli monoecious with bisexual receptacles, simple or branched and its conceptacles are unisexual (Womersley, 1987). Though nutrient uptake and nutrient enrichment of various species of seaweeds, including some Sargassum spp. have been extensively studied (Coutinho and Zingmark, 1993; Pérez-Mayorga et al., 2011; Perini and Bracken, 2014, Reef et al., 2012, Schaffelke and Klumpp, 1998), there is no information available on the impacts of ammonium $\left(\mathrm{NH}_{4}-\mathrm{N}\right)$ and phosphate $\left(\mathrm{PO}_{4}{ }^{3-}-\mathrm{P}\right)$ supplementation on $S$. podacanthum productivity, particularly when cultured in $\mathrm{K}^{+}$-fortified ISW ( $\left.\mathrm{K}^{+} \mathrm{ISW}\right)$.

Nutrients, such as nitrogen $(\mathrm{N})$ and phosphorus $(\mathrm{P})$, are limiting factors for photosynthesis of seaweeds (Larned, 1998). $\mathrm{N}$ is a limiting nutrient in the growth of Sargassum spp. cultured in Hawaii (Larned, 1998) and
Taiwan (Hwang et al., 2004), $\mathrm{P}$ is also considered a limiting factor for $S$. natans and $S$. fluitans growth in the western North Atlantic (Lapointe, 1986). As nutrient limitation on the growth of seaweed is species dependent (Larned, 1998), the majority of seaweed species grow faster in ammonium-enriched than in phosphate-enriched media (Larned, 1998). Supplying $\left(\mathrm{NH}_{4}-\mathrm{N}\right)$ is more efficient than nitrate $\left(\mathrm{NO}_{3}^{-}-\mathrm{N}\right)$ for seaweed growth (Atkinson and Smith, 1983). Thus, the combined $\mathrm{NH}_{4}-\mathrm{N}$ and phosphate $\left(\mathrm{PO}_{4}{ }^{3-}-\mathrm{P}\right)$ has a stronger effect on the growth of $S$. baccularia than a single nutrient (Schaffelke and Klumpp, 1998). However, the information on the impacts of nutrient supplementation during $S$. podacanthum culture, in $\mathrm{K}^{+} \mathrm{ISW}$, is lacking. The present study aims to examine the effects of different $\mathrm{N}$ and $\mathrm{P}$ concentrations, through the supplementation of $\mathrm{NH}_{4}-\mathrm{N}$ and $\mathrm{PO}_{4}{ }^{3-}-\mathrm{P}$, on the growth of $S$. podacanthum in $\mathrm{K}^{+} \mathrm{ISW}$ of WA under the laboratory conditions.

\section{Materials and Methods}

\section{Sargassum podacanthum Collection}

The entire thallus length of $S$. podacanthum, identified by WA Herbarium, were hand-picked from Point Peron, WA (latitude $32^{\circ} 16.3 \mathrm{~S}$, longitude $115^{\circ}$ 41.2E) and immediately transported in OW containers to Curtin Aquatic Research Laboratory (CARL). The $S$. podacanthum was acclimated in aerated $\mathrm{OW}$ at $22^{\circ} \mathrm{C}$ in three $114 \mathrm{~L}$ aquaria, under a down-welling photo-lux density of $120 \mu \mathrm{mol}$ photon $\mathrm{m}^{-2} \mathrm{~s}^{-1}$ and 14:10 h light: dark cycle (Hanisak and Samuel, 1987). The thalli were washed to remove all epiphytes and then rested one more day in aerated OW. The middle of the thalli at the same developmental stage were cut to pieces of approximately $3,500 \mathrm{mg}$ each and then immediately transferred into the cultured media.

\section{Experimental Setup}

The 45 ppt ISW was procured from a lake in Wannamal, WA $\left(31^{\circ} 15 \mathrm{~S}, 116^{\circ} 05 \mathrm{E}\right)$ and brought to CARL and kept in 10,000L reservoir. The ISW and fresh water were filtered through a $0.5 \mu \mathrm{m}$ glass fibre membrane and then mixed to achieve $35 \mathrm{ppt}$ water. At the salinity of $35 \mathrm{ppt}$, the $\left[\mathrm{K}^{+}\right]$in $\mathrm{OW}$ and ISW was, respectively, 351.1 and $84.4 \mathrm{mg} \mathrm{L}^{-1}$; therefore $508.5 \mathrm{mg} \mathrm{L}^{-1}$ of anhydrous potassium chloride was added to ISW to achieve the $\left[\mathrm{K}^{+}\right]$in ISW equivalent to the $\left[\mathrm{K}^{+}\right]$in $\mathrm{OW}$. The OW at 35 ppt was sourced from Hillary Habour $\left(31^{\circ} 83 \mathrm{~S}\right.$, $115^{\circ} 74 \mathrm{E}$ ) and stored at CARL and was also filtered through a $0.5 \mu \mathrm{m}$ glass fibre membrane before using in the trial.

A total of fifty-two (52) 1.5 L-glass beakers filled with $1 \mathrm{~L}$ of water were used for the 84 day-trial. The 
growth of the $S$. podacanthum was determined at five levels of nutrient concentrations in $\mathrm{OW}$ and $\mathrm{K}^{+} \mathrm{ISW}$. The nutrients were provided as molar $\mathrm{NH}_{4}-\mathrm{N}: \mathrm{PO}_{4}{ }^{3-}-\mathrm{P}$ equal to $10: 1$ by the weekly addition ammonium chloride $\left(\mathrm{NH}_{4} \mathrm{Cl}\right)$ and sodium dihydrogen phosphate $\left(\mathrm{NaH}_{2} \mathrm{PO}_{4}\right)$ mixtures (Campbell, 2001). Five different concentrations of $\mathrm{NH}_{4}-\mathrm{N}: \mathrm{PO}_{4}{ }^{3-}-\mathrm{P}$ were $80: 8,120: 12$, 160:16, 200:20 and 240:24 $\mu \mathrm{M}$ (Liu et al., 2004). The needed amount of $\mathrm{NH}_{4} \mathrm{Cl}$ and $\mathrm{NaH}_{2} \mathrm{PO}_{4}$ for $\mathrm{NH}_{4}$ $\mathrm{N}: \mathrm{PO}_{4}{ }^{3-}-\mathrm{P}$ 80:8, 120:12, 160:16, 200:20 and 240:24 $\mu \mathrm{M}$ were, respectively, 4.28 and $0.96,6.42$ and $1.44,8.56$ and $1.92,10.70$ and $2.40,12.84$ and $2.88 \mathrm{mg}$, weighed and stirred to dilute in a part of the cultured media which were taken out from the cultured beakers. The waters were then returned back to the beakers and diluted in the $1 \mathrm{~L}$ cultured medium by a small glass stick. Thirteen treatments were set up in four replicates (including five nutrient concentrations in OW (OW_80, OW_120, OW_160, OW_200, OW_240), five nutrient concentrations ${ }^{-}$in $\mathrm{K}^{+} \overline{\mathrm{ISW}}$ (ISW_ $\overline{\mathrm{S}} 8$, ISW 120, ISW 160, ISW 200, ISW 240) and the controls of ambient OW (OW_0), ambient $\mathrm{K}^{+} \mathrm{ISW}$ (ISW_0) and a raw ISW at 35 ppt.

The salinity was maintained at $34-35$ ppt by adding freshwater to compensate for any losses due to evaporation throughout the trial. All beakers were directly exposed under the white fluorescent lights of $90 \mu$ mol photon $\mathrm{m}^{-2} \mathrm{~s}^{-1}$ on a 14:10 h light:dark cycle and ambient room temperature of $20-26^{\circ} \mathrm{C}$ (Hanisak and Samuel, 1987).

\section{Data Collection}

Water quality parameters of the culture media and the fresh biomass of $S$. podacanthum were measured every 14 days and at the commencement and termination of the trial.

Nitrate $\left(\mathrm{NO}_{3}{ }^{-} \mathrm{N}\right)$ was measured by the Cadmium Reduction Method (Method 8171 for $0-5 \mathrm{mg} \mathrm{L}^{-1}$ and Method 8039 for higher concentrations). Nitrite $\left(\mathrm{NO}_{2}^{-}-\right.$ N) was measured by the Diazotization Method (Method 8507 for concentration less than $0.350 \mathrm{mg} \mathrm{L}^{-1}$ ) and the Ferrous Sulfate Method (Method 8153 for concentration higher than $\left.0.350 \mathrm{mg} \mathrm{L}^{-1}\right)$. Ammonium $\left(\mathrm{NH}_{4}-\mathrm{N}\right)$ was measured by the Salicylate Method (Method 8155 for concentration less than $0.05 \mathrm{mg} \mathrm{L}^{-1}$; Method 10023 for concentration higher than $0.05 \mathrm{mg} \mathrm{L}^{-1}$ ). Phosphate $\left(\mathrm{PO}_{4}{ }^{3-}-\mathrm{P}\right)$ was measured by the Amino Acid Method (Method 8178 for $0-30 \mathrm{mg} \mathrm{L}^{-1}$ ). These parameters were determined by using a Hach DR/890 handheld meter (Hach, Loveland, Colorado, USA). Total Kjeldahl Nitrogen (TKN) was measured according to the Official Method of the AOAC (Helrich, 1990) (method 937.48) by analysing nitrogen using Kjeltec Auto 1030 analyser (Foss Tecator, Höganäs, Sweden).

All $S$. podacanthum thalli were removed from the beakers by a small net and then dried by soft hand towels
(Ahmad et al., 2011). The thalli were quickly transferred to a mechanical weighing scale (Model GX-4000, A\&D Company Limited, Tokyo, Japan). After weighing, the thalli were immediately returned back to the cultured media. At the commencement, three pieces of $S$. podacanthum which were the same developmental stage and similar parts of the $S$. podacanthum thalli were used for culturing in the trial were weighed and placed on aluminium foil trays to dry in an air oven at $60^{\circ} \mathrm{C}$ for 72 $\mathrm{h}$ to get dried biomass (McDermid and Stuercke, 2003). The ratio of dried/fresh biomass were applied to calculate the dried biomass of the $S$. podacanthum throughout the trial. All the fresh $S$. podacanthum by the termination was weighed and dried similarly.

The cumulative specific growth rates (SGR) was calculated as: $\mu_{a}=\left(\ln A_{t}-\ln A_{o}\right) \times 100 / t$. Where: $\mu_{a}$ was the SGR of $S$. podacanthum $\left(\% \mathrm{~d}^{-1}\right) ; A_{t}$ and $A_{o}$ were the dried weights (mg) of the total $S$. podacanthum biomass at a current time $(t, d)$ and the commencement of the trial $(0, d) ; t$ was the current time of the trial (days).

Every day at 9-11 AM, the salinity was recorded by a portable refractometer (RHS-10ATC, Xiamen Ming Xin Instrument, Xiamen, Fujian, China) and the $\mathrm{pH}$ was recorded by a pH metre (CyberScan pH 300, Eutech Instrument, Singapore).

\section{Data Analysis}

All data were analysed using SPSS for Windows version 24.0. The Levene's test was used to test the homoscedasticity of variances of the samples. When the homoscedasticity of the variances of the samples was violated, the translog or square root transformation was undertaken before proceeding with the variance test. The significant differences at $\mathrm{p}<0.05$ among the means of variables were compared by using Analysis of Variance (ANOVA), pair samples t-test and Least Significant Difference (LSD) post hoc tests. Correlation analysis was used to determine relationship among variables.

\section{Results}

\section{Biomass of Sargassum podacanthum}

Although the standing biomass of the $S$. podacanthum varied as the time progressed, different nutrient enrichments resulted in significant $(\mathrm{p}<0.05)$ differences in the growth of $S$. podacanthum. All $S$. podacanthum in ambient ISW (control) died after the first 14 days. The standing biomass of $S$. podacanthum increased with increased nutrient supplementation concentrations. The ratio 160:16 resulted in a significantly $(\mathrm{p}<0.05)$ higher $S$. podacanthum standing biomass than the $S$. podacanthum exposed to all other nutrient concentrations throughout the trial. 
Table 1: Dried biomass (mg) of $S$. podacanthum at control and five supplementation concentrations of $\mathrm{NH}_{4}-\mathrm{N}: \mathrm{PO}_{4}{ }^{3-}-\mathrm{P}$ in $\mathrm{Ocean}$ Water $(\mathrm{OW})$ and $\mathrm{K}^{+}$-fortifed Inland Saline Water ( $\left.\mathrm{K}^{+} \mathrm{ISW}\right)$

\begin{tabular}{|c|c|c|c|c|c|c|c|}
\hline Time & Water & Control & $80: 8$ & $120: 12$ & $160: 16$ & $200: 20$ & $240: 24$ \\
\hline \multirow[t]{2}{*}{ Day 1} & OW & $441.39 \pm 3.49$ & $442.63 \pm 10.36$ & $451.33 \pm 4.32$ & $445.73 \pm 3.10$ & $446.36 \pm 2.89$ & $449.46 \pm 7.16$ \\
\hline & $\mathrm{K}^{+} \mathrm{ISW}$ & $445.11 \pm 9.84$ & $439.83 \pm 16.75$ & $439.83 \pm 13.97$ & $443.25 \pm 10.35$ & $443.25 \pm 11.26$ & $445.11 \pm 3.56$ \\
\hline \multirow[t]{2}{*}{ Day 14} & OW & $472.76 \pm 3.88$ & $493.26 \pm 15.84$ & $457.23 \pm 26.55$ & $515.00 \pm 21.33$ & $448.84 \pm 34.71$ & $506.93 \pm 29.87$ \\
\hline & $\mathrm{K}^{+} \mathrm{ISW}$ & $477.11 \pm 13.86^{\mathrm{bc}}$ & $423.37 \pm 22.78^{\mathrm{bc}}$ & $486.11 \pm 37.54^{b}$ & $566.88 \pm 43.09^{\mathrm{a}}$ & $403.18 \pm 32.26^{\mathrm{c}}$ & $490.15 \pm 27.59^{\mathrm{ab}}$ \\
\hline \multirow[t]{2}{*}{ Day 28} & OW & $492.95 \pm 37.11$ & $491.719 \pm 44.55$ & $539.54 \pm 51.40$ & $568.437 \pm 33.48$ & $479.28 \pm 63.04$ & ${ }_{1} 569.67 \pm 31.71$ \\
\hline & $\mathrm{K}^{+} \mathrm{ISW}$ & $464.37 \pm 8.66^{\mathrm{b}}$ & $422.44 \pm 20.02^{\mathrm{b}}$ & $514.69 \pm 36.14^{\mathrm{ab}}$ & $601.66 \pm 43.48^{\mathrm{a}}$ & $403.80 \pm 37.64^{b}$ & ${ }_{2} 492.64 \pm 28.49^{\mathrm{ab}}$ \\
\hline \multirow[t]{2}{*}{ Day 42} & OW & $481.77 \pm 44.91$ & ${ }_{1} 492.64 \pm 44.84$ & ${ }_{1} 600.42 \pm 57.46$ & $604.77 \pm 42.64$ & ${ }_{1} 506.62 \pm 82.57$ & ${ }_{1} 593.59 \pm 42.32$ \\
\hline & $\mathrm{K}^{+} \mathrm{ISW}$ & $444.80 \pm 27.35^{\mathrm{b}}$ & ${ }_{2} 387.03 \pm 39.91^{\mathrm{b}}$ & ${ }_{2} 468.10 \pm 38.01^{\mathrm{b}}$ & $635.21 \pm 59.00^{\mathrm{a}}$ & ${ }_{2} 409.08 \pm 33.39^{\mathrm{b}}$ & ${ }_{2} 422.75 \pm 22.27^{\mathrm{b}}$ \\
\hline \multirow[t]{2}{*}{ Day 56} & OW & $432.69 \pm 58.20^{\mathrm{b}}$ & ${ }_{1} 545.13 \pm 57.77^{\mathrm{ab}}$ & ${ }_{1} 683.36 \pm 66.35^{\mathrm{a}}$ & $617.82 \pm 42.82^{\mathrm{a}}$ & ${ }_{1} 576.82 \pm 53.20^{\mathrm{ab}}$ & ${ }_{1} 620.30 \pm 39.67^{\mathrm{a}}$ \\
\hline & $\mathrm{K}^{+} \mathrm{ISW}$ & $437.35 \pm 46.05^{\mathrm{b}}$ & ${ }_{2} 355.97 \pm 44.22^{\mathrm{b}}$ & ${ }_{2} 468.11 \pm 45.24^{\mathrm{ab}}$ & $619.68 \pm 58.70^{\mathrm{a}}$ & ${ }_{2} 371.19 \pm 52.48^{\mathrm{b}}$ & ${ }_{2} 444.49 \pm 47.70^{\mathrm{b}}$ \\
\hline \multirow[t]{2}{*}{ Day 70} & OW & $403.18 \pm 54.33^{b}$ & $465.30 \pm 20.39^{b}$ & ${ }_{1}^{6} 689.57 \pm 111.90^{\mathrm{a}}$ & $647.95 \pm 64.84^{\mathrm{a}}$ & ${ }_{1} 610.05 \pm 43.83^{\mathrm{a}}$ & $575.57 \pm 62.18^{\mathrm{ab}}$ \\
\hline & $\mathrm{K}^{+} \mathrm{ISW}$ & $381.13 \pm 13.18^{\mathrm{ab}}$ & $331.74 \pm 42.97^{\mathrm{b}}$ & ${ }_{2} 392.00 \pm 79.77^{\mathrm{ab}}$ & $535.50 \pm 27.06^{\mathrm{a}}$ & ${ }_{2} 295.40 \pm 105.67^{\mathrm{b}}$ & $403.18 \pm 20.84^{\mathrm{ab}}$ \\
\hline \multirow[t]{2}{*}{ Day 84} & OW & $366.84 \pm 57.45^{\mathrm{b}}$ & $393.55 \pm 12.25_{\mathrm{bc}}$ & ${ }_{1} 585.82 \pm 63.37^{\mathrm{a}}$ & $563.77 \pm 49.53^{\mathrm{a}}$ & 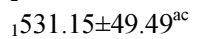 & ${ }_{1} 525.56 \pm 76.90^{\mathrm{ac}}$ \\
\hline & $\mathrm{K}^{+} \mathrm{ISW}$ & $354.10 \pm 15.63^{\mathrm{ab}}$ & $286.70 \pm 31.34^{\mathrm{b}}$ & ${ }_{2} 332.36 \pm 64.39 \mathrm{ab}$ & $448.22 \pm 16.55^{\mathrm{a}}$ & ${ }_{2} 265.27 \pm 99.90^{\mathrm{b}}$ & ${ }_{2} 335.47 \pm 20.93^{\mathrm{ab}}$ \\
\hline
\end{tabular}

Values (mean $\pm \mathrm{SE}$ ) within a row sharing a common superscript are not significantly different (LSD test; $\mathrm{p}>0.05 ; \mathrm{n}=4)$. Values (mean $\pm \mathrm{SE}$ ) within a column at a time sharing a common subscript are not the significantly different at $\mathrm{p}<0.05(\mathrm{t}$-test, $\mathrm{n}=4)$

In OW, $S$. podacanthum showed a significantly $(p<0.05)$ higher biomass in OW 120, OW 160, OW 200 than the other nutrient concentrations in the second half of the trial period and reached the maximum biomass at day 70 and then declined (Table 1). In $\mathrm{K}^{+} \mathrm{ISW}$, the $S$. podacanthum biomass was significantly higher in ISW 160 throughout the trial and the biomass at the end was similar to that at the beginning. However, at all other nutrient concentrations, the biomass did not change during the first 70 days and then significantly declined (Table 1).

Two water types (OW or $\mathrm{K}^{+} \mathrm{ISW}$ ) did not show any effect on the standing biomass of $S$. podacanthum in the first 28 days, but significantly $(\mathrm{p}<0.05)$ affected the $S$. podacanthum biomass from day 42 of the trial period. Furthermore, higher concentrations of nutrients were significantly correlated with the standing biomass of $S$. podacanthum. From the day 56 until the end of the trial, the standing biomass of $S$. podacanthum was significantly higher in $\mathrm{OW}$ than in $\mathrm{K}^{+} \mathrm{ISW}$ at high nutrient concentrations (120:12, 200:20, 240:24), except when enriched with $\mathrm{NH}_{4}-\mathrm{N}: \mathrm{PO}_{4}{ }^{3-}-\mathrm{P}$ 160:16 $\mu \mathrm{M}$. At water with no nutrient supplementation and the ratio of 160:16, the standing biomass of $S$. podacanthum grown in OW showed no significant differences from that of $S$. podacanthum grown in $\mathrm{K}^{+} \mathrm{ISW}$.

The biomass of $S$. podacanthum was significantly correlated $\left(\mathrm{R}^{2}>0.7, \mathrm{p}<0.05\right)$ with time (in fortnights) (Table 2), water temperature and the concentrations of $\mathrm{NO}_{2}{ }^{-}-\mathrm{N}, \mathrm{NH}_{4}-\mathrm{N}, \mathrm{TKN}$ and $\mathrm{PO}_{4}{ }^{3-}-\mathrm{P}$ in waters (Table 3). The SGR of the $S$. podacanthum decreased towards the end of the trial and varied significantly $(\mathrm{p}<0.5)$ with the nutrient levels in two water types (Table 4). The SGR was significantly correlated with the water $\mathrm{pH}$, temperature and water quality parameters, with the exception of $\mathrm{NO}_{3}^{-}-\mathrm{N}$ (Table 3). The SGR ranged from negative values to $1.70 \% \mathrm{~d}^{-1}$, recorded in the first 14 days in ISW 160. The $S$. podacanthum SGR was significantly $(\overline{\mathrm{p}}<0.01)$ affected by the water types from day 42 onwards, except at the waters without nutrient supplementation and the ratio of 160:16.

At OW 120 and OW 240, the SGR of $S$. podacanthum remained positive during the entire trial. No effects of nutrient enrichments on SGR of $S$. podacanthum were observed in the first 42 days, however, from the day 42 , the higher nutrient enrichment resulted in significantly $(\mathrm{p}<0.05)$ higher SGR.

A negative SGR of $S$. podacanthum was recorded in ISW 80, ISW 200 and ISW 240, as the trial progressed. In other nutrient levels in $\overline{\mathrm{K}}^{+}$ISW the SGR gradually decreased from the beginning to the end of the trial. An exception SGR data were recorded at ISW_160, where highest $\quad(p<0.05) \quad$ SGR among five nutrient concentrations over time were observed and showed the only positive SGR of the $S$. podacanthum in $\mathrm{K}^{+} \mathrm{ISW}$ during the whole trial (Table 4).

\section{Water Quality}

The $\mathrm{pH}$ of the OW was similarly in five different nutrient concentrations and control during the trial. However, from the day 42 onwards, the $\mathrm{pH}$ in the ISW 240 was significantly $(p<0.05)$ higher than that in all other supplementation levels in $\mathrm{K}^{+} \mathrm{ISW}$ (Fig. 1a). The temperature of the culture media was similar in all the nutrient concentrations, ranging from 20 to $26^{\circ} \mathrm{C}$ (Fig. 1b).

At the commencement of the trial, the $\mathrm{NO}_{2}^{-}-\mathrm{N}, \mathrm{NO}_{3}^{-}-\mathrm{N}$, $\mathrm{NH}_{4}-\mathrm{N}, \mathrm{TKN}$ and $\mathrm{PO}_{4}{ }^{3-}-\mathrm{P}$ concentrations in raw ISW were similar in OW 0 and ISW 0. After two weeks, the concentrations of $\mathrm{NO}_{2}^{-}-\mathrm{N}, \mathrm{TKN}$ and $\mathrm{PO}_{4}{ }^{3-}-\mathrm{P}$ significantly $(\mathrm{p}<0.05)$ increased while $\mathrm{NO}_{3}^{-}-\mathrm{N}$ and $\mathrm{NH}_{4}-\mathrm{N}$ remained unchanged in raw ISW.

The $[\mathrm{N}]$ remained unchanged in the early stages of the trial and significantly $(p<0.05)$ increased by the end of the trial in both water types. During the trial, $\left[\mathrm{NO}_{2}^{-}-\mathrm{N}\right]$ was similar at all the nutrient levels. Both $\mathrm{NO}_{2}^{-}-\mathrm{N}$ and $\mathrm{NO}_{3}^{-}-\mathrm{N}$ varied widely in $\mathrm{K}^{+} \mathrm{ISW}$, but in ISW $160, \mathrm{NO}_{2}^{-}-\mathrm{N}$ was stable as the time progressed (Table ${ }^{-}$), while $\mathrm{NO}_{3}{ }^{-} \mathrm{N}$ decreased significantly by the end of the trial (Table 6). 
Table 2: The regression correlation of the $S$. podacanthum biomass in $\mathrm{mg}(\mathrm{y})$ with the time in fortnight $(\mathrm{x})$ in Ocean Water (OW) and $\mathrm{K}^{+}$fortified Inland Saline Water $\left(\mathrm{K}^{+} \mathrm{ISW}\right)$ at control and five supplementation concentrations of $\mathrm{NH}_{4}-\mathrm{N}: \mathrm{PO}_{4}{ }^{3-}-\mathrm{P}(\mu \mathrm{M})$

\begin{tabular}{llll}
\hline Water & $\mathrm{NH}_{4}-\mathrm{N}_{\mathrm{PO}}{ }^{3-}-\mathrm{P}$ & Regression & $\mathrm{R}^{2}$ \\
\hline OW & Control & $\mathrm{y}=-7.89 \mathrm{x}^{2}+48.02 \mathrm{x}+407.40$ & $\mathrm{R}^{2}=0.94$ \\
& $80: 8$ & $\mathrm{y}=-10.71 \mathrm{x}^{2}+80.38 \mathrm{x}+367.69$ & $\mathrm{R}^{2}=0.77$ \\
& $120: 12$ & $\mathrm{y}=-10.53 \mathrm{x}^{2}+120.39 \mathrm{x}+301.52$ & $\mathrm{R}^{2}=0.82$ \\
$160: 16$ & $\mathrm{y}=-11.08 \mathrm{x}^{2}+112.51 \mathrm{x}+337.68$ & $\mathrm{R}^{2}=0.94$ \\
& $200: 20$ & $\mathrm{y}=-3.66 \mathrm{x}^{2}+53.34 \mathrm{x}+373.94$ & $\mathrm{R}^{2}=0.74$ \\
& $240: 24$ & $\mathrm{y}=-12.73 \mathrm{x}^{2}+116.69 \mathrm{x}+336.53$ & $\mathrm{R}^{2}=0.97$ \\
$\mathrm{~K}^{+}$ISW & Control & $\mathrm{y}=-4.98 \mathrm{x}^{2}+11.94 \mathrm{x}+422.08$ & $\mathrm{R}^{2}=0.93$ \\
& $80: 8$ & $\mathrm{y}=-2.98 \mathrm{x}^{2}-1.50 \mathrm{x}+443.74$ & $\mathrm{R}^{2}=0.99$ \\
& $120: 12$ & $\mathrm{y}=-12.07 \mathrm{x}^{2}+77.30 \mathrm{x}+377.8$ & $\mathrm{R}^{2}=0.93$ \\
& $160: 16$ & $\mathrm{y}=-20.80 \mathrm{x}^{2}+165.37 \mathrm{x}+304.67$ & $\mathrm{R}^{2}=0.98$ \\
& $200: 20$ & $\mathrm{y}=-4.98 \mathrm{x}^{2}+11.94 \mathrm{x}+422.08$ & $\mathrm{R}^{2}=0.93$ \\
& $240: 24$ & $\mathrm{y}=-7.14 \mathrm{x}^{2}+37.42 \mathrm{x}+426.48$ & $\mathrm{R}^{2}=0.86$ \\
\hline
\end{tabular}

Table 3: Pearson correlation of $S$. podacanthum biomass and water quality parameters $(\mathrm{N}=48)$

\begin{tabular}{llllllll}
\hline Dependent variable & $\mathrm{pH}$ & Temperature & $\mathrm{NO}_{2}{ }^{-}-\mathrm{N}$ & $\mathrm{NO}_{3}{ }^{-}-\mathrm{N}$ & $\mathrm{PO}_{4}{ }^{3-}-\mathrm{P}$ & $\mathrm{NH}_{4}-\mathrm{N}$ & $\mathrm{TKN}$ \\
\hline Biomass & 0.012 & $0.226^{* *}$ & $-0.365^{* *}$ & $-0.121^{*}$ & $-0.273^{* *}$ & $-0.110^{*}$ & $-0.197^{* *}$ \\
SGR & $-0.157^{* *}$ & $0.163^{* *}$ & $-0.234^{* *}$ & -0.115 & $-0.134^{*}$ & $-0.207^{* *}$ & $-0.630^{* *}$ \\
\hline
\end{tabular}

$(* *)-$ Correlation is significant at the 0.01 level (2-tailed); $(*)$ - Correlation is significant at the 0.05 level (2-tailed)

Table 4: Specific growth rate $\left(\% \mathrm{~d}^{-1}\right)$ of $S$. podacanthum at control and five supplementation concentrations of $\mathrm{NH}_{4}-\mathrm{N}^{-}: \mathrm{PO}_{4}{ }^{3-}-\mathrm{P}$ in Ocean Water $(\mathrm{OW})$ and $\mathrm{K}^{+}$-fortifed Inland Saline Water $\left(\mathrm{K}^{+} \mathrm{ISW}\right)$

\begin{tabular}{|c|c|c|c|c|c|c|c|}
\hline Time & Water & Control & $80: 8$ & $120: 12$ & $160: 16$ & $200: 20$ & $240: 24$ \\
\hline \multirow[t]{2}{*}{ Day 1-14 } & $\mathrm{OW}$ & $0.49 \pm 0.08$ & $0.77 \pm 0.22$ & $0.06 \pm 0.45$ & $1.01 \pm 0.27$ & $-0.03 \pm 0.54$ & $0.83 \pm 0.43$ \\
\hline & $\mathrm{K}^{+} \mathrm{ISW}$ & $0.49 \pm 0.22^{\mathrm{b}}$ & $-0.29 \pm 0.27^{\mathrm{bc}}$ & $0.66 \pm 0.64^{\mathrm{ab}}$ & $1.70 \pm 0.64^{\mathrm{a}}$ & $-0.74 \pm 0.53^{c}$ & $0.66 \pm 0.45^{\mathrm{ab}}$ \\
\hline \multirow[t]{2}{*}{ Day 1-28 } & OW & $0.36 \pm 0.26$ & $0.34 \pm 0.29$ & $0.59 \pm 0.32$ & $0.85 \pm 0.20$ & $0.15 \pm 0.49$ & $0.83 \pm 0.19$ \\
\hline & $\mathrm{K}^{+} \mathrm{ISW}$ & $0.15 \pm 0.13^{\mathrm{bc}}$ & $-0.15 \pm 0.21^{b c}$ & $0.54 \pm 0.18^{\mathrm{ab}}$ & $1.06 \pm 0.31^{\mathrm{a}}$ & $-0.38 \pm 0.30^{\mathrm{c}}$ & $0.35 \pm 0.23^{\mathrm{abc}}$ \\
\hline \multirow[t]{2}{*}{ Day 1-42 } & OW & $0.18 \pm 0.21$ & $0.23 \pm 0.18$ & $0.65 \pm 0.21$ & $0.71 \pm 0.16$ & $0.19 \pm 0.42$ & ${ }_{1} 0.65 \pm 0.16^{\mathrm{a}}$ \\
\hline & $\mathrm{K}^{+} \mathrm{ISW}$ & $-0.01 \pm 0.12^{\mathrm{b}}$ & $-0.34 \pm 0.28^{b}$ & $0.13 \pm 0.18^{\mathrm{b}}$ & $0.83 \pm 0.25^{\mathrm{a}}$ & $-0.21 \pm 0.15^{\mathrm{b}}$ & $2^{-0.13 \pm 0.13^{b}}$ \\
\hline \multirow[t]{2}{*}{ Day 1-56 } & OW & $-0.08 \pm 0.22^{b}$ & ${ }_{1} 0.34 \pm 0.19^{\mathrm{ab}}$ & ${ }_{1} 0.72 \pm 0.17^{\mathrm{a}}$ & $0.57 \pm 0.12^{\mathrm{a}}$ & ${ }_{1} 0.43 \pm 0.16^{\mathrm{ab}}$ & ${ }_{1} 0.57 \pm 0.11^{\mathrm{a}}$ \\
\hline & $\mathrm{K}^{+} \mathrm{ISW}$ & $-0.06 \pm 0.18^{\mathrm{bc}}$ & $2^{-}-0.41 \pm 0.26^{\mathrm{c}}$ & ${ }_{2} 0.16 \pm 0.19^{\mathrm{ab}}$ & $0.57 \pm 0.18^{\mathrm{a}}$ & $2^{-0.37 \pm 0.26^{b c}}$ & $2^{-0.03 \pm 0.18^{\mathrm{bc}}}$ \\
\hline \multirow[t]{2}{*}{ Day 1-70 } & oW & $-0.16 \pm 0.17^{b}$ & ${ }_{1} 0.07 \pm 0.09^{\mathrm{ab}}$ & ${ }_{1} 0.54 \pm 0.19^{\mathrm{a}}$ & $0.50 \pm 0.16^{\mathrm{a}}$ & ${ }_{1} 0.42 \pm 0.10^{\mathrm{a}}$ & ${ }_{1} 0.32 \pm 0.16^{\mathrm{ab}}$ \\
\hline & $\mathrm{K}^{+} \mathrm{ISW}$ & $-0.22 \pm 0.02^{\mathrm{ab}}$ & ${ }_{2}-0.42 \pm 0.21^{\mathrm{b}}$ & ${ }_{2}-0.27 \pm 0.37^{b}$ & $0.26 \pm 0.09^{\mathrm{a}}$ & $2^{-0.19 \pm 0.15^{b}}$ & $2^{-0.14} \pm 0.07^{\mathrm{ab}}$ \\
\hline \multirow[t]{2}{*}{ Day 1-84 } & OW & $-0.27 \pm 0.17^{\mathrm{b}}$ & $-0.14 \pm 0.12^{\mathrm{bc}}$ & ${ }_{1} 0.30 \pm 0.12^{\mathrm{a}}$ & $0.27 \pm 0.11^{\mathrm{ac}}$ & ${ }_{1} 0.20 \pm 0.11^{\mathrm{ac}}$ & $0.15 \pm 0.19^{\mathrm{abc}}$ \\
\hline & $\mathrm{K}^{+} \mathrm{ISW}$ & $-0.28 \pm 0.06^{\mathrm{ab}}$ & $-0.54 \pm 0.16^{\mathrm{b}}$ & $2^{-0.43 \pm 0.30^{b}}$ & $0.01 \pm 0.06^{\mathrm{a}}$ & $2^{-0.32 \pm 0.18^{\mathrm{ab}}}$ & $2^{-0.35 \pm 0.08^{\mathrm{ab}}}$ \\
\hline
\end{tabular}

Values (mean \pm SE) within a row sharing a common superscript are not significantly different (LSD test; $p>0.05 ; n=4)$. Values $($ mean $\pm \mathrm{SE}$ ) within a column at a time sharing a common subscript are not the significantly different at $\mathrm{p}<0.05(\mathrm{t}$-test, $\mathrm{n}=4)$

Table 5: The concentrations of $\mathrm{NO}_{2}^{-}-\mathrm{N}$ in water cultured $S$. podacanthum at control and five supplementation concentrations of $\mathrm{NH}_{4-} \mathrm{N}: \mathrm{PO}_{4}{ }^{3-}-\mathrm{P}$ in Ocean Water $(\mathrm{OW})$ and $\mathrm{K}^{+}$-fortifed Inland Saline Water $\left(\mathrm{K}^{+} \mathrm{ISW}\right)$

\begin{tabular}{|c|c|c|c|c|c|c|c|}
\hline Time & Water & Control & $80: 8$ & $120: 12$ & $160: 16$ & $200: 20$ & $240: 24$ \\
\hline \multirow[t]{2}{*}{$\overline{\text { Day } 1}$} & $\mathrm{OW}$ & $0.011 \pm 0.001^{\mathrm{a}}$ & $0.007 \pm 0.000^{b}$ & $0.006 \pm 0.000^{b}$ & $0.007 \pm 0.000^{b}$ & $0.007 \pm 0.000^{b}$ & $0.006 \pm 0.000^{b}$ \\
\hline & $\mathrm{K}^{+} \mathrm{ISW}$ & $0.012 \pm 0.000^{\mathrm{bc}}$ & $0.012 \pm 0.000^{\mathrm{bc}}$ & $0.014 \pm 0.000^{\mathrm{a}}$ & $0.011 \pm 0.002^{b}$ & $0.015 \pm 0.001^{\mathrm{a}}$ & $0.007 \pm 0.001^{\mathrm{d}}$ \\
\hline \multirow[t]{2}{*}{ Day 14} & OW & $0.011 \pm 0.001^{\mathrm{b}}$ & $0.007 \pm 0.001^{\mathrm{c}}$ & $0.007 \pm 0.001^{\mathrm{c}}$ & $0.012 \pm 0.001^{b}$ & $0.028 \pm 0.001^{\mathrm{a}}$ & $0.008 \pm 0.001^{\mathrm{c}}$ \\
\hline & $\mathrm{K}^{+} \mathrm{ISW}$ & $0.008 \pm 0.001^{\mathrm{b}}$ & $0.015 \pm 0.001^{\mathrm{a}}$ & $0.010 \pm 0.000^{\mathrm{b}}$ & $0.013 \pm 0.000^{\mathrm{a}}$ & $0.010 \pm 0.001^{\mathrm{b}}$ & $0.008 \pm 0.000^{\mathrm{b}}$ \\
\hline \multirow[t]{2}{*}{ Day 28} & OW & $0.007 \pm 0.000^{\mathrm{b}}$ & $0.007 \pm 0.000^{\mathrm{b}}$ & $0.007 \pm 0.001^{\mathrm{b}}$ & $0.009 \pm 0.000^{\mathrm{ab}}$ & $0.010 \pm 0.001^{\mathrm{a}}$ & $0.011 \pm 0.002^{\mathrm{a}}$ \\
\hline & $\mathrm{K}^{+} \mathrm{ISW}$ & $0.012 \pm 0.002^{\mathrm{b}}$ & $0.008 \pm 0.000^{\mathrm{d}}$ & $0.013 \pm 0.000^{\mathrm{b}}$ & $0.011 \pm 0.001^{b c}$ & $0.009 \pm 0.001^{\mathrm{cd}}$ & $0.026 \pm 0.001^{\mathrm{a}}$ \\
\hline \multirow[t]{2}{*}{ Day 42} & OW & $0.008 \pm 0.000$ & ${ }_{1} 0.009 \pm 0.000$ & $0.006 \pm 0.000$ & $0.008 \pm 0.000$ & $0.008 \pm 0.001$ & $0.010 \pm 0.001$ \\
\hline & $\mathrm{K}^{+} \mathrm{ISW}$ & $0.011 \pm 0.001^{b}$ & ${ }_{2} 0.013 \pm 0.001^{\mathrm{b}}$ & $0.018 \pm 0.002^{\mathrm{a}}$ & $0.015 \pm 0.001^{\mathrm{a}}$ & $0.010 \pm 0.001^{b}$ & $0.016 \pm 0.001^{\mathrm{a}}$ \\
\hline \multirow[t]{2}{*}{ Day 56} & OW & $0.004 \pm 0.001^{\mathrm{bc}}$ & ${ }_{1} 0.007 \pm 0.002^{\mathrm{a}}$ & $0.004 \pm 0.001^{b c}$ & $0.006 \pm 0.002^{\mathrm{ab}}$ & $0.003 \pm 0.001^{b c}$ & $0.002 \pm 0.002^{\mathrm{c}}$ \\
\hline & $\mathrm{K}^{+} \mathrm{ISW}$ & $0.022 \pm 0.005^{\mathrm{b}}$ & ${ }_{2} 0.013 \pm 0.001^{\mathrm{c}}$ & $0.008 \pm 0.003^{\mathrm{d}}$ & $0.014 \pm 0.002^{\mathrm{c}}$ & $0.031 \pm 0.004^{\mathrm{a}}$ & $0.002 \pm 0.001^{\mathrm{e}}$ \\
\hline \multirow[t]{2}{*}{ Day 70} & OW & $0.020 \pm 0.000^{\mathrm{a}}$ & $0.007 \pm 0.000^{\mathrm{b}}$ & $0.007 \pm 0.000^{\mathrm{b}}$ & $0.006 \pm 0.000^{\mathrm{b}}$ & $0.006 \pm 0.001^{b}$ & $0.005 \pm 0.001^{\mathrm{b}}$ \\
\hline & $\mathrm{K}^{+} \mathrm{ISW}$ & $0.018 \pm 0.002^{b}$ & $0.009 \pm 0.000^{\mathrm{d}}$ & $0.019 \pm 0.005^{\mathrm{b}}$ & $0.013 \pm 0.001^{\mathrm{c}}$ & $0.044 \pm 0.006^{\mathrm{a}}$ & $0.005 \pm 0.000^{\mathrm{e}}$ \\
\hline \multirow[t]{2}{*}{ Day 84} & OW & $0.033 \pm 0.003^{\mathrm{a}}$ & $0.011 \pm 0.002^{\mathrm{c}}$ & $0.015 \pm 0.003^{b c}$ & $0.006 \pm 0.002^{\mathrm{d}}$ & $0.020 \pm 0.005^{b}$ & $0.007 \pm 0.000^{\mathrm{d}}$ \\
\hline & $\mathrm{K}^{+} \mathrm{ISW}$ & $0.038 \pm 0.002^{\mathrm{a}}$ & $0.026 \pm 0.002^{b}$ & $0.016 \pm 0.001^{\mathrm{c}}$ & $0.014 \pm 0.002^{c}$ & $0.041 \pm 0.003^{\mathrm{a}}$ & $0.013 \pm 0.001^{\mathrm{c}}$ \\
\hline
\end{tabular}

Values (mean \pm SE) within a row sharing a common superscript are not significantly different (LSD test; $p>0.05 ; n=4)$. Values $($ mean $\pm \mathrm{SE}$ ) within a column at a time sharing a common subscript are not the significantly different at $\mathrm{p}<0.05(\mathrm{t}$-test, $\mathrm{n}=4$ ) 
Table 6: The concentrations of $\mathrm{NO}_{3}^{-}-\mathrm{N}$ in water cultured $S$. podacanthum at control and five supplementation concentrations of $\mathrm{NH}_{4}-\mathrm{N}: \mathrm{PO}_{4}{ }^{3-}-\mathrm{P}$ in Ocean Water $(\mathrm{OW})$ and $\mathrm{K}^{+}$-fortifed Inland Saline Water $\left(\mathrm{K}^{+} \mathrm{ISW}\right)$

\begin{tabular}{|c|c|c|c|c|c|c|c|}
\hline Time & Water & Control & $80: 8$ & $120: 12$ & $160: 16$ & 200:20 & $240: 24$ \\
\hline \multirow[t]{2}{*}{ Day 1} & OW & $12.17 \pm 0.08^{b}$ & ${ }_{1} 3.50 \pm 0.04^{\mathrm{a}}$ & $1^{2.47 \pm 0.06^{\mathrm{c}}}$ & ${ }_{1} 1.67 \pm 0.06^{\mathrm{d}}$ & ${ }_{1} 1.83 \pm 0.02^{\mathrm{e}}$ & ${ }_{1} 1.47 \pm 0.02^{f}$ \\
\hline & $\mathrm{K}^{+} \mathrm{ISW}$ & $2.37 \pm 0.06^{\mathrm{ac}}$ & ${ }_{2} 2.27 \pm 0.02^{\mathrm{a}}$ & ${ }_{2} 1.73 \pm 0.08^{\mathrm{b}}$ & $2.30 \pm 0.07^{\mathrm{c}}$ & $2.47 \pm 0.02^{\mathrm{a}}$ & ${ }_{2} 2.27 \pm 0.05^{\mathrm{c}}$ \\
\hline \multirow[t]{2}{*}{ Day 14} & OW & $2.07 \pm 0.13^{\mathrm{bc}}$ & $2.63 \pm 0.06^{\mathrm{ab}}$ & $1.90 \pm 0.11^{\mathrm{c}}$ & $2.90 \pm 0.23^{\mathrm{a}}$ & $2.37 \pm 0.15^{\mathrm{b}}$ & ${ }_{1} 1.83 \pm 0.10^{\mathrm{c}}$ \\
\hline & $\mathrm{K}^{+} \mathrm{ISW}$ & $1.87 \pm 0.10^{\mathrm{d}}$ & $2.60 \pm 0.11^{\mathrm{bc}}$ & $2.83 \pm 0.13^{\mathrm{b}}$ & $2.07 \pm 0.10^{\mathrm{cd}}$ & ${ }_{2} 3.63 \pm 0.22^{\mathrm{a}}$ & ${ }_{2} 2.40 \pm 0.12^{\mathrm{c}}$ \\
\hline \multirow[t]{2}{*}{ Day 28} & OW & ${ }_{1} 2.10 \pm 0.37^{b}$ & $1.93 \pm 0.10^{\mathrm{b}}$ & $2.33 \pm 0.06^{\mathrm{b}}$ & $2.23 \pm 0.19^{\mathrm{b}}$ & ${ }_{1} 1.77 \pm 0.06^{\mathrm{b}}$ & ${ }_{1} 2.90 \pm 0.19^{\mathrm{a}}$ \\
\hline & $\mathrm{K}^{+} \mathrm{ISW}$ & $1.47 \pm 0.05^{\mathrm{d}}$ & $2.33 \pm 0.12^{\mathrm{bc}}$ & $2.67 \pm 0.25^{\mathrm{b}}$ & $1.93 \pm 0.08^{\mathrm{cd}}$ & ${ }_{2} 3.43 \pm 0.06^{\mathrm{a}}$ & ${ }_{2} 3.90 \pm 0.13^{\mathrm{a}}$ \\
\hline \multirow[t]{2}{*}{ Day 42} & OW & $1.53 \pm 0.19^{\mathrm{bc}}$ & $2.57 \pm 0.66^{\mathrm{a}}$ & $2.33 \pm 0.47^{\mathrm{ab}}$ & ${ }_{1} 1.60 \pm 0.23^{\mathrm{bc}}$ & $1.03 \pm 0.05^{\mathrm{c}}$ & $2.90 \pm 0.23^{\mathrm{a}}$ \\
\hline & $\mathrm{K}^{+} \mathrm{ISW}$ & $2.13 \pm 0.25^{\mathrm{b}}$ & $2.80 \pm 0.16^{\mathrm{b}}$ & $2.00 \pm 0.11^{\mathrm{b}}$ & $2.53 \pm 0.08^{\mathrm{b}}$ & $1.13 \pm 0.06^{\mathrm{c}}$ & $3.63 \pm 0.13^{\mathrm{a}}$ \\
\hline \multirow[t]{2}{*}{ Day 56} & OW & $2.77 \pm 0.16^{\mathrm{b}}$ & $2.73 \pm 0.14^{b}$ & $3.07 \pm 0.62^{\mathrm{b}}$ & $1.80 \pm 0.11^{\mathrm{c}}$ & $5.03 \pm 0.12^{\mathrm{a}}$ & $2.87 \pm 0.37^{b}$ \\
\hline & $\mathrm{K}^{+} \mathrm{ISW}$ & $3.53 \pm 0.22^{\mathrm{bc}}$ & $2.47 \pm 0.13^{\mathrm{d}}$ & $3.93 \pm 0.13^{\mathrm{b}}$ & ${ }_{2}^{3} .97 \pm 0.63^{\mathrm{b}}$ & $5.30 \pm 0.49^{\mathrm{a}}$ & $2.70 \pm 0.15^{\mathrm{cd}}$ \\
\hline \multirow[t]{2}{*}{ Day 70} & OW & ${ }_{1} 2.37 \pm 0.22^{\mathrm{a}}$ & ${ }_{1} 1.80 \pm 0.07^{\mathrm{b}}$ & $2.63 \pm 0.17^{\mathrm{a}}$ & $2.23 \pm 0.08^{\mathrm{ab}}$ & $2.73 \pm 0.08^{\mathrm{a}}$ & ${ }_{1} 1.97 \pm 0.12^{b}$ \\
\hline & $\mathrm{K}^{+} \mathrm{ISW}$ & ${ }_{2} 4.20 \pm 0.37^{\mathrm{a}}$ & ${ }_{2} 3.50 \pm 0.08^{\mathrm{bc}}$ & ${ }_{2} 3.20 \pm 0.12^{\mathrm{b}}$ & ${ }_{2} 3.90 \pm 0.12^{\mathrm{ab}}$ & $2.90 \pm 0.27^{\mathrm{c}}$ & ${ }_{2} 3.13 \pm 0.16^{\mathrm{c}}$ \\
\hline \multirow[t]{2}{*}{ Day 84} & OW & $1.85 \pm 0.39^{\mathrm{cb}}$ & $2.40 \pm 0.15^{\mathrm{b}}$ & ${ }_{1} 3.63 \pm 0.94^{\mathrm{ab}}$ & $1.77 \pm 0.10^{\mathrm{c}}$ & $4.83 \pm 1.25^{\mathrm{a}}$ & $3.47 \pm 0.33^{\mathrm{ab}}$ \\
\hline & $\mathrm{K}^{+} \mathrm{ISW}$ & $2.47 \pm 0.73^{\mathrm{ab}}$ & $2.80 \pm 0.33^{\mathrm{ab}}$ & ${ }_{2} 1.53 \pm 0.17^{\mathrm{c}}$ & $1.03 \pm 0.43^{\mathrm{c}}$ & $6.13 \pm 1.13^{\mathrm{a}}$ & $3.10 \pm 0.08^{\mathrm{b}}$ \\
\hline
\end{tabular}

Values (mean \pm SE) within a row sharing a common superscript are not significantly different (LSD test; $p>0.05 ; n=4)$. Values $($ mean \pm SE) within a column at a time sharing a common subscript are not the significantly different at $\mathrm{p}<0.05(\mathrm{t}$-test, $\mathrm{n}=4$ )

Table 7: The concentrations of $\mathrm{NH}_{4}-\mathrm{N}$ in water cultured $S$. podacanthum at control and five supplementation concentrations of $\mathrm{NH}_{4}-\mathrm{N}: \mathrm{PO}_{4}{ }^{3-}-\mathrm{P}$ in Ocean Water $(\mathrm{OW})$ and $\mathrm{K}^{+}$-fortifed Inland Saline Water $\left(\mathrm{K}^{+} \mathrm{ISW}\right)$

\begin{tabular}{|c|c|c|c|c|c|c|c|}
\hline Time & Water & Control & $80: 8$ & $120: 12$ & $160: 16$ & $200: 20$ & 240:24 \\
\hline \multirow{2}{*}{ Day 1} & OW & Negligible $^{f}$ & ${ }_{1} 0.977 \pm 0.009^{\mathrm{e}}$ & ${ }_{1} 1.717 \pm 0.014^{\mathrm{d}}$ & ${ }_{1} 2.087 \pm 0.005^{c}$ & ${ }_{1} 2.643 \pm 0.005^{b}$ & $2.750 \pm 0.000^{\mathrm{a}}$ \\
\hline & $\mathrm{K}^{+} \mathrm{ISW}$ & Negligible $^{f}$ & ${ }_{2} 1.003 \pm 0.005^{\mathrm{e}}$ & ${ }_{2} 1.617 \pm 0.009^{\mathrm{d}}$ & ${ }_{2} 1.987 \pm 0.010^{\mathrm{c}}$ & ${ }_{2} 2.607 \pm 0.006^{\mathrm{b}}$ & $2.750 \pm 0.000^{\mathrm{a}}$ \\
\hline \multirow[t]{2}{*}{ Day 14} & OW & Negligible & Negligible & Negligible & Negligible & Negligible & Negligible \\
\hline & $\mathrm{K}^{+} \mathrm{ISW}$ & Negligible & Negligible & Negligible & Negligible & Neglig & Negligible \\
\hline \multirow[t]{2}{*}{ Day 28} & OW & Negligible & & $\mathrm{Neg}$ & Negli & Neglig & Neglig \\
\hline & $\mathrm{K}^{+} \mathrm{ISW}$ & Negligib & & & Negligit & $\mathrm{Neq}$ & Neglig \\
\hline \multirow[t]{2}{*}{ Day 42} & OW & 0.002 & .002 & $0.010 \pm 0.007$ & ${ }_{1} 0.010 \pm 0.007$ & ${ }_{1} 0.030 \pm 0.021$ & $0.033 \pm 0.017$ \\
\hline & $\mathrm{K}^{+} \mathrm{ISW}$ & $0.010 \pm 0.004^{\mathrm{c}}$ & $0.040 \pm 0.028^{\mathrm{c}}$ & $0.080 \pm 0.018^{\mathrm{cb}}$ & ${ }_{2} 0.173 \pm 0.062^{\mathrm{ab}}$ & ${ }_{2} 0.193 \pm 0.110^{\mathrm{a}}$ & $0.033 \pm 0.009^{c}$ \\
\hline \multirow[t]{2}{*}{ Day 56} & OW & Negligible $^{\mathrm{b}}$ & ${ }_{1} 0.040 \pm 0.028^{\mathrm{a}}$ & $0.010 \pm 0.007^{\mathrm{b}}$ & Negligible $^{\mathrm{b}}$ & Negligible $^{\mathrm{b}}$ & $0.027 \pm 0.015^{\mathrm{ab}}$ \\
\hline & $\mathrm{K}^{+} \mathrm{ISW}$ & Negligible $^{b}$ & ${ }_{2}$ Negligible ${ }^{\mathrm{b}}$ & Negligible $^{\mathrm{b}}$ & gible $^{b}$ & $0.020 \pm 0.014^{\mathrm{ab}}$ & $0.037 \pm 0.012^{\mathrm{a}}$ \\
\hline \multirow[t]{2}{*}{ Day 70} & OW & ${ }_{1}$ Negligible $^{c}$ & $0.063 \pm 0.023^{\mathrm{b}}$ & ${ }_{1} 0.133 \pm 0.046^{\mathrm{a}}$ & ${ }_{1} 0.093 \pm 0.023^{\mathrm{ab}}$ & $0.043 \pm 0.010^{\mathrm{b}}$ & $0.127 \pm 0.002^{\mathrm{a}}$ \\
\hline & $\mathrm{K}^{+} \mathrm{ISW}$ & ${ }_{2} 0.137 \pm 0.029^{b}$ & $0.080 \pm 0.015^{\mathrm{bc}}$ & ${ }_{2} 0.047 \pm 0.006^{\mathrm{bc}}$ & ${ }_{2} 0.207 \pm 0.010^{\mathrm{a}}$ & $0.035 \pm 0.007^{\mathrm{c}}$ & $0.103 \pm 0.017^{\mathrm{b}}$ \\
\hline \multirow[t]{2}{*}{ Day 84} & OW & $0.053 \pm 1.650$ & ${ }_{1} 0.030 \pm 0.004$ & $0.133 \pm 0.028$ & ${ }_{1} 0.033 \pm 0.013$ & $0.207 \pm 0.104$ & ${ }_{1} 0.073 \pm 0.025$ \\
\hline & $\mathrm{K}^{+} \mathrm{ISW}$ & $0.179 \pm 0.036$ & $0.248 \pm 0.021$ & $0.249 \pm 0.085$ & ${ }_{2} 0.191 \pm 0.067$ & $0.137 \pm 0.015$ & $0.217 \pm 0.017$ \\
\hline
\end{tabular}

Values (mean \pm SE) within a row sharing a common superscript are not significantly different (LSD test; $p>0.05 ; n=4)$. Values $($ mean $\pm \mathrm{SE}$ ) within a column at a time sharing a common subscript are not the significantly different at $\mathrm{p}<0.05(\mathrm{t}$-test, $\mathrm{n}=4)$

Table 8: The concentrations of TKN in water cultured $S$. podacanthum at control and five supplementation concentrations of $\mathrm{NH}_{4}$ $\mathrm{N}: \mathrm{PO}_{4}{ }^{3-}-\mathrm{P}$ in Ocean Water $(\mathrm{OW})$ and $\mathrm{K}^{+}$-fortifed Inland Saline Water $\left(\mathrm{K}^{+} \mathrm{ISW}\right)$

\begin{tabular}{|c|c|c|c|c|c|c|c|}
\hline Time & Water & Control & $80: 8$ & $120: 12$ & $160: 16$ & $200: 20$ & $240: 24$ \\
\hline \multirow[t]{2}{*}{ Day 1} & OW & $0.42 \pm 0.03^{\mathrm{f}}$ & ${ }_{1} 1.07 \pm 0.02^{\mathrm{e}}$ & ${ }_{1} 1.84 \pm 0.04^{\mathrm{d}}$ & ${ }_{1} 2.33 \pm 0.02^{c}$ & $2.71 \pm 0.02^{\mathrm{b}}$ & ${ }_{1} 3.01 \pm 0.06^{\mathrm{a}}$ \\
\hline & $\mathrm{K}^{+} \mathrm{ISW}$ & $0.54 \pm 0.07^{\mathrm{f}}$ & ${ }_{2} 1.80 \pm 0.02^{\mathrm{e}}$ & ${ }_{2} 1.98 \pm 0.04^{\mathrm{d}}$ & $2.17 \pm 0.03^{c}$ & ${ }_{2} 3.39 \pm 0.04^{b}$ & ${ }_{2} 3.60 \pm 0.10^{\mathrm{a}}$ \\
\hline \multirow[t]{2}{*}{ Day 14} & OW & $2.03 \pm 0.06^{\mathrm{c}}$ & $2.45 \pm 0.14^{b}$ & $2.10 \pm 0.06^{\mathrm{bc}}$ & $2.36 \pm 0.05^{\mathrm{bc}}$ & $2.45 \pm 0.09^{\mathrm{b}}$ & ${ }_{1} 3.15 \pm 0.19^{\mathrm{a}}$ \\
\hline & $\mathrm{K}^{+} \mathrm{ISW}$ & $2.36 \pm 0.23^{\mathrm{d}}$ & ${ }_{2} 3.55 \pm 0.07^{\mathrm{b}}$ & $2.47 \pm 0.23^{\mathrm{d}}$ & ${ }_{2} 3.13 \pm 0.06^{\mathrm{c}}$ & ${ }_{2} 4.27 \pm 0.03^{\mathrm{a}}$ & ${ }_{2} 3.74 \pm 0.09^{b}$ \\
\hline \multirow[t]{2}{*}{ Day 28} & OW & ${ }_{1} 0.23 \pm 0.06^{\mathrm{c}}$ & ${ }_{1} 1.12 \pm 0.10^{\mathrm{b}}$ & ${ }_{1} 0.96 \pm 0.07^{b}$ & ${ }_{1} 1.17 \pm 0.16^{\mathrm{b}}$ & $1.31 \pm 0.03^{\mathrm{ab}}$ & ${ }_{1} 1.59 \pm 0.04^{\mathrm{a}}$ \\
\hline & $\mathrm{K}^{+} \mathrm{ISW}$ & ${ }_{2} 1.70 \pm 0.15^{\mathrm{e}}$ & ${ }_{2} 1.70 \pm 0.17^{\mathrm{e}}$ & ${ }_{2} 2.75 \pm 0.17^{\mathrm{c}}$ & ${ }_{2} 2.40 \pm 0.03^{\mathrm{d}}$ & ${ }_{2} 3.10 \pm 0.12^{b}$ & ${ }_{2} 3.31 \pm 0.15^{\mathrm{a}}$ \\
\hline \multirow[t]{2}{*}{ Day 42} & OW & $1.82 \pm 0.21^{\mathrm{b}}$ & $2.57 \pm 0.42^{b}$ & $2.15 \pm 0.29^{b}$ & $2.15 \pm 0.09^{b}$ & ${ }_{1} 4.02 \pm 0.23^{\mathrm{a}}$ & $2.19 \pm 0.34^{b}$ \\
\hline & $\mathrm{K}^{+} \mathrm{ISW}$ & ${ }_{2} 3.74 \pm 0.09^{c}$ & ${ }_{2} 4.53 \pm 0.29^{c}$ & ${ }_{2} 5.09 \pm 0.66^{\mathrm{b}}$ & ${ }_{2} 4.30 \pm 0.14^{\mathrm{c}}$ & ${ }_{2} 7.52 \pm 0.34^{\mathrm{a}}$ & ${ }_{2} 5.14 \pm 0.37^{b}$ \\
\hline \multirow[t]{2}{*}{ Day 56} & OW & ${ }_{1}^{0} 0.37 \pm 0.03^{\mathrm{d}}$ & $2.29 \pm 0.44^{\mathrm{cd}}$ & ${ }_{1} 1.59 \pm 0.09^{c}$ & $1.96 \pm 0.23^{\mathrm{bc}}$ & $2.80 \pm 0.41^{\mathrm{b}}$ & $4.02 \pm 0.23^{\mathrm{a}}$ \\
\hline & $\mathrm{K}^{+} \mathrm{ISW}$ & ${ }_{2} 3.27 \pm 0.26^{\mathrm{c}}$ & ${ }_{2} 3.31 \pm 0.23^{\mathrm{c}}$ & ${ }_{2} 3.74 \pm 0.18^{\mathrm{c}}$ & ${ }_{2} 4.76 \pm 0.06^{\mathrm{b}}$ & ${ }_{2} 5.18 \pm 0.66^{\mathrm{b}}$ & ${ }_{2} 6.54 \pm 0.43^{\mathrm{a}}$ \\
\hline \multirow[t]{2}{*}{ Day 70} & OW & ${ }_{1} 1.59 \pm 0.09^{\mathrm{bc}}$ & $4.11 \pm 0.34^{\mathrm{ab}}$ & $5.51 \pm 0.69^{\mathrm{a}}$ & ${ }_{1} 1.54 \pm 0.21^{\mathrm{c}}$ & $2.94 \pm 0.21^{\mathrm{b}}$ & ${ }_{1} 0.61 \pm 0.17^{\mathrm{c}}$ \\
\hline & $\mathrm{K}^{+} \mathrm{ISW}$ & ${ }_{2} 3.27 \pm 0.09^{b}$ & $4.25 \pm 0.14^{\mathrm{ab}}$ & $5.56 \pm 0.52^{\mathrm{a}}$ & ${ }_{2}^{4} 4.81 \pm 0.18^{\mathrm{a}}$ & ${ }_{2} 4.11 \pm 0.41^{\mathrm{ab}}$ & ${ }_{2} 4.76 \pm 1.45^{\mathrm{ab}}$ \\
\hline \multirow[t]{2}{*}{ Day 84} & OW & ${ }_{1} 1.88 \pm 0.46^{\mathrm{c}}$ & ${ }_{1} 3.41 \pm 0.36^{\mathrm{ab}}$ & ${ }_{1} 3.41 \pm 0.60^{\mathrm{ab}}$ & ${ }_{1} 3.02 \pm 0.37^{\mathrm{bc}}$ & $3.36 \pm 0.55^{\mathrm{ab}}$ & $4.76 \pm 0.66^{\mathrm{a}}$ \\
\hline & $\mathrm{K}^{+} \mathrm{ISW}$ & ${ }_{2} 4.61 \pm 0.49^{b}$ & ${ }_{2} 6.76 \pm 0.30^{\mathrm{a}}$ & ${ }_{2} 5.26 \pm 0.73 b$ & ${ }_{2} 4.82 \pm 0.73^{b}$ & $4.25 \pm 0.26^{\mathrm{b}}$ & $5.18 \pm 0.21^{\mathrm{b}}$ \\
\hline
\end{tabular}

Values (mean $\pm \mathrm{SE}$ ) within a row sharing a common superscript are not significantly different (LSD test; $p>0.05 ; n=4)$. Values $($ mean $\pm \mathrm{SE}$ ) within a column at a time sharing a common subscript are not the significantly different at $\mathrm{p}<0.05(\mathrm{t}-\mathrm{test}, \mathrm{n}=4)$ 
Table 9: The concentrations of $\mathrm{PO}_{4}{ }^{3-}-\mathrm{P}$ in water cultured $S$. podacanthum at control and five supplementation concentrations of $\mathrm{NH}_{4}-\mathrm{N}: \mathrm{PO}_{4}{ }^{3-}-\mathrm{P}$ in Ocean Water $(\mathrm{OW})$ and $\mathrm{K}^{+}$-fortifed Inland Saline Water $\left(\mathrm{K}^{+} \mathrm{ISW}\right)$

\begin{tabular}{|c|c|c|c|c|c|c|c|}
\hline Time & Water & Control & $80: 8$ & $120: 12$ & $160: 16$ & $200: 20$ & $240: 24$ \\
\hline \multirow[t]{2}{*}{$\overline{\text { Day } 1}$} & OW & $1.00 \pm 0.00^{\mathrm{c}}$ & $2.57 \pm 0.05^{b}$ & ${ }_{1} 2.50 \pm 0.12^{b}$ & $2.70 \pm 0.07^{\mathrm{ab}}$ & ${ }_{1} 2.47 \pm 0.02^{b}$ & ${ }_{1} 2.83 \pm 0.02^{\mathrm{a}}$ \\
\hline & $\mathrm{K}^{+} \mathrm{ISW}$ & $1.07 \pm 0.02^{\mathrm{c}}$ & $2.80 \pm 0.04^{\mathrm{b}}$ & $2.67 \pm 0.02^{\mathrm{b}}$ & $2.60 \pm 0.00^{\mathrm{b}}$ & ${ }_{2} 3.17 \pm 0.02^{\mathrm{a}}$ & ${ }_{2} 3.27 \pm 0.05^{\mathrm{a}}$ \\
\hline \multirow[t]{2}{*}{ Day 14} & OW & ${ }_{1} 1.03 \pm 0.02^{\mathrm{b}}$ & ${ }_{1} 1.17 \pm 0.02^{\mathrm{a}}$ & ${ }_{1} 1.10 \pm 0.04^{\mathrm{b}}$ & ${ }_{1} 0.63 \pm 0.02^{\mathrm{c}}$ & ${ }_{1}^{0.83 \pm 0.02^{\mathrm{c}}}$ & ${ }_{1} 0.83 \pm 0.02^{\mathrm{c}}$ \\
\hline & $\mathrm{K}^{+} \mathrm{ISW}$ & ${ }_{2} 1.20 \pm 0.04^{\mathrm{b}}$ & ${ }_{2} 1.30 \pm 0.04^{\mathrm{a}}$ & ${ }_{2} 1.00 \pm 0.04^{\mathrm{d}}$ & ${ }_{2} 1.30 \pm 0.04^{\mathrm{a}}$ & ${ }_{2} 1.10 \pm 0.04^{\mathrm{c}}$ & ${ }_{2} 1.23 \pm 0.02^{\mathrm{b}}$ \\
\hline \multirow[t]{2}{*}{ Day 28} & OW & ${ }_{1} 0.93 \pm 0.08^{\mathrm{ab}}$ & $1.10 \pm 0.07^{\mathrm{a}}$ & $0.97 \pm 0.13^{\mathrm{ab}}$ & ${ }_{1} 0.67 \pm 0.02^{\mathrm{bc}}$ & $1_{1} 0.60 \pm 0.11^{\mathrm{c}}$ & ${ }_{1} 0.83 \pm 0.02^{\mathrm{b}}$ \\
\hline & $\mathrm{K}^{+} \mathrm{ISW}$ & $1.17 \pm 0.02^{\mathrm{ab}}$ & $1.30 \pm 0.04^{\mathrm{a}}$ & $0.87 \pm 0.13^{\mathrm{c}}$ & $1.30 \pm 0.04^{\mathrm{a}}$ & $0.97 \pm 0.08^{\mathrm{bc}}$ & $1.23 \pm 0.02^{\mathrm{a}}$ \\
\hline \multirow[t]{2}{*}{ Day 42} & OW & $0.30 \pm 0.11^{\mathrm{b}}$ & $0.33 \pm 0.02^{b}$ & ${ }_{1} 0.37 \pm 0.13^{\mathrm{b}}$ & ${ }_{1} 0.33 \pm 0.08^{\mathrm{b}}$ & ${ }_{1}^{0.57 \pm 0.02^{\mathrm{b}}}$ & ${ }_{1} 1.23 \pm 0.06^{\mathrm{a}}$ \\
\hline & $\mathrm{K}^{+} \mathrm{ISW}$ & $0.50 \pm 0.22^{\mathrm{cd}}$ & $0.27 \pm 0.06^{\mathrm{d}}$ & ${ }_{2} 0.77 \pm 0.10^{\mathrm{bc}}$ & $0.83 \pm 0.02^{\mathrm{b}}$ & $1.03 \pm 0.02^{\mathrm{b}}$ & $1.53 \pm 0.05^{\mathrm{a}}$ \\
\hline \multirow[t]{2}{*}{ Day 56} & OW & $0.60 \pm 0.11$ & $0.90 \pm 0.12$ & $0.97 \pm 0.08$ & $0.53 \pm 0.06$ & $0.77 \pm 0.18$ & $0.57 \pm 0.10$ \\
\hline & $\mathrm{K}^{+} \mathrm{ISW}$ & $1.03 \pm 0.17^{\mathrm{ab}}$ & $1.13 \pm 0.20^{\mathrm{ab}}$ & $1.07 \pm 0.18^{\mathrm{ab}}$ & $1.27 \pm 0.16^{\mathrm{a}}$ & ${ }_{2} 1.10 \pm 0.45^{\mathrm{ab}}$ & $0.77 \pm 0.02^{\mathrm{b}}$ \\
\hline \multirow[t]{2}{*}{ Day 70} & OW & $0.47 \pm 0.02$ & $0.73 \pm 0.08$ & ${ }_{1} 0.43 \pm 0.05$ & $0.60 \pm 0.11$ & $0.50 \pm 0.04$ & $0.43 \pm 0.08$ \\
\hline & $\mathrm{K}^{+} \mathrm{ISW}$ & $1.13 \pm 0.02^{\mathrm{bc}}$ & $0.63 \pm 0.02^{\mathrm{c}}$ & $1.37 \pm 0.17^{\mathrm{b}}$ & $0.87 \pm 0.33^{c}$ & $2.53 \pm 0.63^{\mathrm{a}}$ & $0.57 \pm 0.02^{\mathrm{c}}$ \\
\hline \multirow[t]{2}{*}{ Day 84} & OW & $1.17 \pm 0.33^{\mathrm{ab}}$ & $1.27 \pm 0.02^{\mathrm{ab}}$ & $1.43 \pm 0.34^{\mathrm{ab}}$ & $1.30 \pm 0.32^{\mathrm{ab}}$ & $1.87 \pm 0.46^{\mathrm{a}}$ & $1.07 \pm 0.17^{\mathrm{b}}$ \\
\hline & $\mathrm{K}^{+} \mathrm{ISW}$ & $1.43 \pm 0.05^{\mathrm{bc}}$ & $1.90 \pm 0.15^{\mathrm{b}}$ & $1.23 \pm 0.25^{\mathrm{bc}}$ & $1.43 \pm 0.06^{\mathrm{cb}}$ & ${ }_{2} 3.50 \pm 0.36^{\mathrm{a}}$ & $0.93 \pm 0.08^{\mathrm{c}}$ \\
\hline
\end{tabular}

Values (mean \pm SE) within a row sharing a common superscript are not significantly different (LSD test; $p>0.05 ; n=4$ ). Values (mean \pm SE) within a column at a time sharing a common subscript are not the significantly different at $\mathrm{p}<0.05(\mathrm{t}$-test, $\mathrm{n}=4$ )
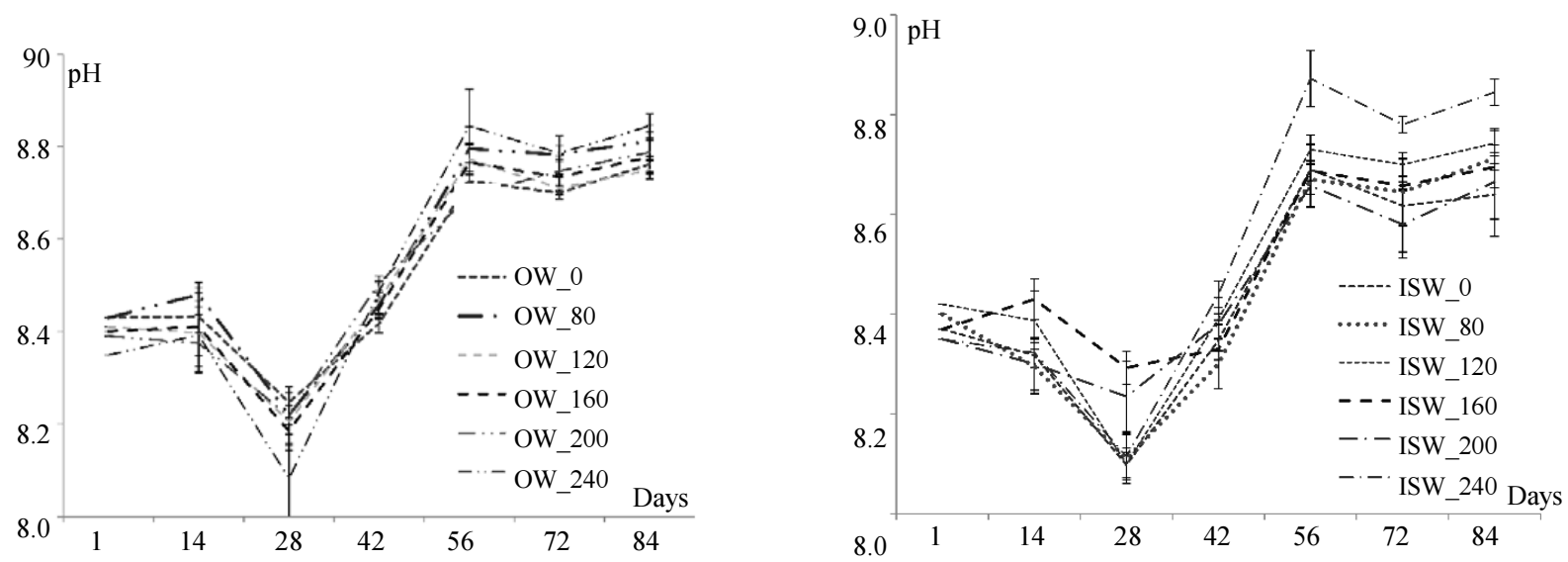

(a)
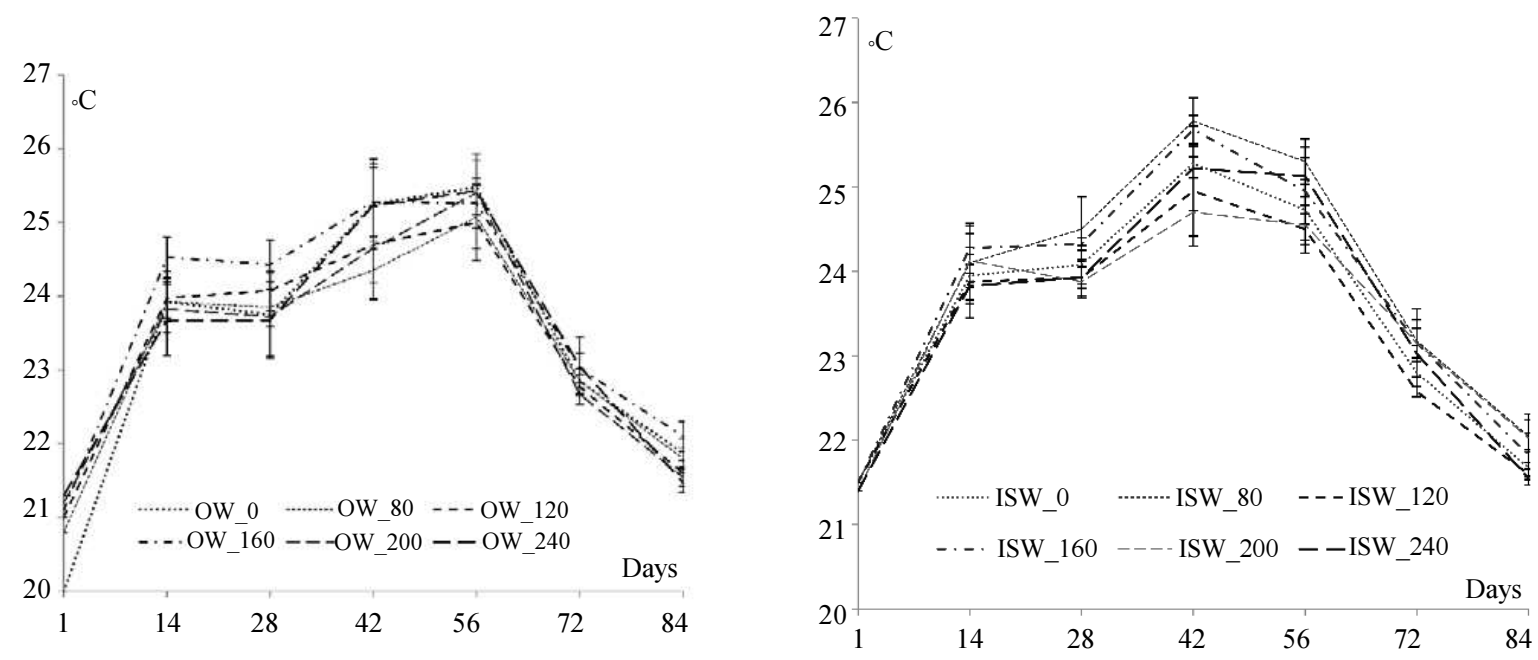

(b)

Fig.1: The $\mathrm{pH}$ (a) and temperature (b) of the water cultured $S$. podacanthum at controls and five supplement concentrations of $\mathrm{NH}_{4}$ $\mathrm{N}: \mathrm{PO}_{4}{ }^{3-}-\mathrm{P} 80: 8,120: 12,160: 16,200: 20$ and 240:24 $\mu \mathrm{M}$ were respectively supplied to ocean water (OW_0, OW_80, OW_120, OW_160, OW_200, OW_240) and $\mathrm{K}^{+}$-fortified inland saline water (ISW_0, ISW_80, ISW_120, ISW_ 160 , ISW_200, ISW_240) 
The $\mathrm{NH}_{4}-\mathrm{N}$ and TKN concentrations significantly rose with increasing nutrient enrichment levels and were higher in $\mathrm{K}^{+} \mathrm{ISW}$ than in OW. However, after releasing $S$. podacanthum into the water, $\left[\mathrm{NH}_{4}-\mathrm{N}\right]$ was approximately negligible over the first 56 days, then increased to a maximum of $0.25 \mathrm{mg} \mathrm{L}^{-1}$, which was lower than at the commencement of the trial (Table 7). Conversely, TKN decreased to a minimal value at day 28 and significantly increased by the end of the trial (Table 8).

The $\left[\mathrm{PO}_{4}{ }^{3-}-\mathrm{P}\right]$ in both water types decreased significantly $(\mathrm{p}<0.05)$ during the trial compared than at the beginning of the trial. It was higher in $\mathrm{K}^{+} \mathrm{ISW}$ than OW at all nutrient supplementation concentrations greater than 80:8 (Table 9).

\section{Discussion}

Taking advantage of the short-seasonal growth of Sargassum (Martin-Smith, 1993), farming Sargassum in salt-affected farms can provide several agricultural uses including by-product for cattle feed (Huisman, 2000). As nutrient requirements of Sargassum spp. in ISW have not yet been researched, the results of this study can be significant in improving technical feasibility of Sargassum culture in ISW. The result of this study has shown that ammonium and phosphate enrichment plays an important role for growing $S$. podacanthum in $\mathrm{K}^{+} \mathrm{ISW}$ under laboratory conditions.

The range of $\mathrm{N}: \mathrm{P}$ atomic ratio of Sargassum spp. is 20:1 to 38:1 (Atkinson and Smith, 1983), while the average N:P for seaweed is from 10:1 to 30:1 (Atkinson and Smith, 1983) and the N:P (in moles) in OW is 37:1 on average (Downing, 1997). The nutrient supplementation $\mathrm{NH}_{4}-\mathrm{N}: \mathrm{PO}_{4}{ }^{3-}-\mathrm{P}$ ratio of $10: 1$ was adapted from similar research of Schaffelke and Klumpp (1998) and Schaffelke (1999), where the N and P demand for $S$. baccularia is from $2.9-15.0$ and 0.10 $0.68 \mu \mathrm{mol} \mathrm{g}{ }^{-1}$ dry weight per day in August to December (Schaffelke and Klumpp, 1998). The $S$. everve grow faster in $\mathrm{NH}_{4}-\mathrm{N} 200 \mu \mathrm{M}$ than in $80 \mu \mathrm{M}$ enriched $\mathrm{OW}$ (Liu et al., 2004), which were the basis level nutrient supplementation for this study. In the media where the $\mathrm{K}^{+}$ISW was enriched with $\mathrm{NH}_{4}-\mathrm{N}$ and $\mathrm{PO}_{4}{ }^{3-}-\mathrm{P}$, the growth of $S$. podacanthum was significantly correlated with the nutrient concentrations. The effect of nutrients within the range 120:12-160:16 $\mu \mathrm{M}$ was visible after one month of cultivation. These nutrient levels resulted in higher and sustainable growth of $S$. podacanthum.

The $S$. podacanthum died in raw ISW after one fortnight reconfirmed the need of $\mathrm{K}^{+}$fortification in ISW for acceptable growth of Sargassum. The $\left[\mathrm{K}^{+}\right]$in ISW significantly affected the growth of $S$. linearifolium, which reached an optimal growth in $\mathrm{K}^{+} \mathrm{ISW}$ at a similar concentration of $\mathrm{K}^{+}$in $\mathrm{OW}$ (Bui et al., 2017b). Therefore, the nutrients were enriched into the $\mathrm{K}^{+} \mathrm{ISW}$ at the similar concentration of $\mathrm{K}^{+}$in the $\mathrm{OW} . \mathrm{K}^{+}$is essential for the growth of plants (Blumwald et al.,
2000; Talling, 2010), particularly for seaweed, as it is recognised as an important internal cation (Kirst, 1977) playing a role in protein and starch synthesis and metabolic processes in living cells (Evans and Sorger, 1966). Moreover, $\mathrm{K}^{+}$balances the osmotic gradient in aquatic plant cells (Malhotra and Glass, 1995) and maintains a standard cellular sodium to potassium ratio (Blumwald et al., 2000). At $35 \mathrm{ppt}$, the $\left[\mathrm{K}^{+}\right]$in ISW is less than one-third of the $\left[\mathrm{K}^{+}\right]$in OW and this low level caused the total mortality of the $S$. podacanthum after two weeks of cultivation even though the $\mathrm{N}$ and $\mathrm{P}$ in raw ISW were similar to ISW_0. When the $S$. podacanthum died due to lack of $\mathrm{K}^{+}$, nutrients were not consumed, so that the $\mathrm{NO}_{3}{ }^{-} \mathrm{N}$ and $\mathrm{NH}_{4}-\mathrm{N}$ concentrations in raw ISW did not change during the first two weeks of culture and the data from this treatment was not continued collected.

The Sargassum's growth and development is seasonal (Schaffelke and Klumpp, 1998; Vuki and Price, 1994) and varies according to the species (McCourt, 1984). The fertile receptacles of the temperate Sargassum are shed in summer and the tropical Sargassum is abundant in winter (McCourt, 1984). The Australian Sargassum, S. tenerrimum, S. fissifolium, S. olygocystum achieve higher biomass and maximum length in summer (December-February), followed by a late summer peak in reproduction (March-May). $S$. linearifolium, on the other hand, achieves its peak in size in June-September and reproduction in SeptemberJanuary (Martin-Smith, 1993). The growth of $S$. podacanthum in $\mathrm{OW}$ and $\mathrm{K}^{+} \mathrm{ISW}$, without any nutrient supplementation resulted in similar life cycles as any other sub-tropical and/or temperate Sargassum spp. Our trial, lasted from September to December and the growths of $S$. podacanthum significantly increased in the first month and then decreased from late October. Wherein the $S$. linearifolium maximum growth occurrs during the late winter and early spring (August-October) and then starts to decline in November (Martin-Smith, 1994), which is also similar to $S$. linearifolium grown under the laboratory conditions (Bui et al., 2017b). In the nutrient enriched condition, the growing stage of $S$. podacanthum lasted up to 70 days, similar to $S$. linearifolium in the natural environment. This result demonstrated that the seasonal growth cycle of $S$. podacanthum in the laboratory condition was similar to the natural out-door conditions.

Temperature and $\mathrm{pH}$ play a crucial role in the growth of Sargassum (Chen and Zou, 2014; Choi et al., 2009). The water temperature, with no significant differences among water types, was $20-26^{\circ} \mathrm{C}$, without any controlled mechanism in place. This temperature range reflected similar OW temperatures during this season (https://www.seatemperature.org/australiapacific/australia/western- australia/, downloaded 23 Dec 2016). According to Hanisak and Samuel (1987), this is a suitable temperature for the maximum growth of Sargassum spp. The correlation of the $S$. 
podacanthum biomass (in the form of a standing crop) and temperature in the present study is similar to $S$. polysystum, S. binderi and S. siliquosum in the natural environment (May-Lin and Ching-lee, 2013). Although the $\mathrm{pH}$ of the culture media was in a suitable range for seaweed growth (Lignell and Pedersén, 1989), pH was lowest in the second fortnight in controls and the nutrient enrichment concentrations of 80:8, 120:12 and 200:20 in both water types, that coincided with the occurrence of Sargassum mortality (Tucker and D'Abramo, 2008). In contrast, $S$. podacanthum grew well in ISW_160, where the $\mathrm{pH}$ was relatively stable over time.

The maximal SGR of $S$. podacanthum in the present study was $1.7 \% \mathrm{~d}^{-1}$ in the first fortnight in the ISW_160, lower than the SGR $\left(4.7 \% \mathrm{~d}^{-1}\right)$ of $S$. horneri (Turner) C. Agardh in the natural OW environment under similar temperatures (Gao and Hua, 1997, Yamauchi, 1984). The growth of Sargassum spp. are species-specific (Hanisak and Samuel, 1987) as $S$. baccularia reaches twice its growth in a $\mathrm{NH}_{4}-\mathrm{N}: \mathrm{PO}_{4}{ }^{3-}-\mathrm{P}$ ratio of $10: 1$, at 3-5 $\mu \mathrm{M} \mathrm{NH}_{4}-\mathrm{N}$ supplied continuously in $\mathrm{OW}$ and the growth rate is reduced when $\mathrm{NH}_{4}-\mathrm{N}$ and $\mathrm{PO}_{4}{ }^{3-}-\mathrm{P}$ are supplemented beyond these ranges (Schaffelke and Klumpp, 1998). The SGR $8 \% \mathrm{~d}^{-1}$ of $S$. baccularia in nutrient enrichment OW (Schaffelke and Klumpp, 1998) is higher than that of $S$. podacanthum from this study in both $\mathrm{OW}$ and $\mathrm{K}^{+} \mathrm{ISW}$, again highlighting the different nutrient requirements among various species of Sargassum. At a similar concentration of N (including $\mathrm{NO}_{3}^{-}-\mathrm{N}$ and $\mathrm{NH}_{4}-\mathrm{N}$ ), the SGR of $S$. podacanthum in OW in the first month and in ISW 160 in the first five fortnights was around $0.3 \% \mathrm{~d}^{-1}$, which is similar to the SGR of the adult stage of S. muticum under natural OW on the floating raft from August to May at Higashiura, where the temperature changed from 25 to $10^{\circ} \mathrm{C}$ and the $\mathrm{NO}_{3}{ }^{-} \mathrm{N}$ is from $1.0-4.1 \mu \mathrm{M}, \mathrm{NH}_{4}-\mathrm{N}$ is from 5.8$11.0 \mu \mathrm{M}$ and $\mathrm{PO}_{4}{ }^{3-}-\mathrm{P}$ is from $0.12-0.35 \mu \mathrm{M}$ for five months (Yamauchi, 1984).

The standing biomass of $S$. podacanthum was significantly correlated to the nutrient levels in the culture media. In addition to the weekly-supplied nutrients, the $\mathrm{N}$ and $\mathrm{P}$ in water were also generated by the decomposition process of dying $S$. podacanthum. The soluble $\mathrm{N}$ and $\mathrm{P}$ concentrations in water are difficult to stabilise and measure as they are quickly cycled by living microbes (Downing, 1997). N and $\mathrm{P}$ have been consumed at different rates (Smith et al., 1986), for example, at the same concentrations, $\mathrm{NH}_{4}-\mathrm{N}$ uptake is faster than $\mathrm{PO}_{4}{ }^{3-}-\mathrm{P}$ (Wallentinus, 1984). S. podacanthum and bacteria in water quickly consumed the provided $\mathrm{PO}_{4}{ }^{3-}-\mathrm{P}$ and $\mathrm{NH}_{4}-\mathrm{N}$, resulting in a similar concentration of $\mathrm{PO}_{4}{ }^{3-}-\mathrm{P}$ in cultured media to that found in natural $\mathrm{OW}$ throughout the trial, particularly in ISW_160, and $\mathrm{NH}_{4}-\mathrm{N}$ quickly reduced to negligible levels after enrichment. The $\left[\mathrm{PO}_{4}{ }^{3-}-\mathrm{P}\right]$ in the culture media was lowest from day 42 to day 70 of cultivation, that was found to be correlated to a high biomass of $S$. podacanthum in both water types. Towards the end of the trial, when a reduction of the $S$. podacanthum biomass was recorded, the $\mathrm{PO}_{4}{ }^{3-}-\mathrm{P}$ and $\mathrm{NH}_{4}-\mathrm{N}$ supplements were not totally consumed and in turn, resulted in the increase of $\mathrm{PO}_{4}{ }^{3-}-\mathrm{P}$ and $\mathrm{NH}_{4}-\mathrm{N}$. A sharp increase in $\mathrm{PO}_{4}{ }^{3-}-\mathrm{P}$ and $\mathrm{NO}_{3}{ }^{-} \mathrm{N}$ in ISW 200 towards the end of the trial was associated with the highest reduction in the $S$. podacanthum biomass. The additional source of organic nitrogen was came from the decomposing dead Sargassum (Robards et al., 1994), therefore, the higher TKN concentration in $\mathrm{K}^{+} \mathrm{ISW}$ than in OW resulted from the higher mortality of $S$. podacanthum in $\mathrm{K}^{+} \mathrm{ISW}$. The present study confirmed that the main factor for the highest standing biomass of $S$. podacanthum in real-time was limited by $\mathrm{N}$, represented by $\mathrm{NO}_{3}{ }^{-}-\mathrm{N}$ and $\mathrm{NO}_{2}^{-}-\mathrm{N}$ rather than $\mathrm{NH}_{4}-\mathrm{N}$, as seaweed can consume $\mathrm{NO}_{3}^{-}-\mathrm{N}$ instead of $\mathrm{NH}_{4}-\mathrm{N}$ when $\mathrm{NH}_{4}-\mathrm{N}$ is insufficient (Jie et al., 2008) or is lower than $0.135 \mathrm{mg} \mathrm{L}^{-1}$ (Balode et al., 1998). During the present trial, $\mathrm{NH}_{4}-\mathrm{N}$ was usually negligible and $\left[\mathrm{NO}_{3}{ }^{-}-\right.$ $\mathrm{N}$ ] was always available at $2-4 \mathrm{mg} \mathrm{L}^{-1}$, which met the requirements of $S$. podacanthum. The $\left[\mathrm{NO}_{3}^{-}-\mathrm{N}\right]$ in water was found to be significantly correlated with the $S$. podacanthum biomass, given that $\left[\mathrm{NO}_{3}{ }^{-} \mathrm{-N}\right]$ and the biomass of $S$. podacanthum were both unchanged at all nutrient levels in the first half of the trial. The increase of $\left[\mathrm{NO}_{3}^{-}-\mathrm{N}\right]$ towards the end of the trial resulted in a reduction of $S$. podacanthum biomass in both water types. In ISW_160, $\left[\mathrm{NO}_{3}{ }^{-}-\mathrm{N}\right]$ was stable over the first four fortnights and then increased, indicating that the $S$. podacanthum biomass decreased after reaching its maximum biomass in the fourth fortnight.

The enrichment of $\mathrm{NH}_{4}-\mathrm{N}$ and $\mathrm{PO}_{4}{ }^{3-}-\mathrm{P}$ from $120: 12$ to $200: 20$ in OW and 120:12 to $160: 16$ in $\mathrm{K}^{+} \mathrm{ISW}$ in the culture of $S$. podacanthum resulted in a higher growth rate. It was clear from the trial that the nutrient levels lower or higher than the above mentioned reduced the growth of $S$. podacanthum, particularly in $\mathrm{K}^{+} \mathrm{ISW}$ and also caused mortality from the early stages of the culture period. This result supports the claims that high nutrient levels inhibit the growth of $S$. baccularia (Schaffelke and Klumpp, 1998) and S. siliquosum (Diaz-Pulido and McCook, 2005). The present study shows that ISW_160 retained the most suitable water for growing $S$. podacanthum in $\mathrm{K}^{+} \mathrm{ISW}$, when the standing biomass increased until the day 70, the highest among the $\mathrm{K}^{+} \mathrm{ISW}$ waters and the SGR of $S$. podacanthum in ISW_160 in the culture period was the only positive SGR among all nutrient supplementations $\mathrm{K}^{+}$ISW.

\section{Conclusion}

The nutrient enrichment of 160:16 $\mu \mathrm{M}$ of $\mathrm{NH}_{4}$ $\mathrm{N}: \mathrm{PO}_{4}{ }^{3-}-\mathrm{P}$, using $\mathrm{NH}_{4} \mathrm{Cl}$ and $\mathrm{NaH}_{2} \mathrm{PO}_{4}$, in ISW which 
was fortified with $\mathrm{K}^{+}$at similar $\mathrm{K}^{+}$concentration in $\mathrm{OW}$ at the same salinity, results in a similar biomass and SGR of $S$. podacanthum cultured in OW. This nutrient level is the most suitable water for growing $S$. podacanthum in $\mathrm{K}^{+} \mathrm{ISW}$. The $S$. podacanthum growth cycle in the laboratory conditions is similar to the natural conditions, wherein, the maximal growth season is from the late winter to early spring.

\section{Acknowledgement}

We thank Simon Longbottom, Huy Quang Dinh for their help with seaweed collection and assistance in laboratory. Our thanks are also extended to Dr. Thuy Nguyen, Dr. Thanh Ngo and Proof-reading-service.com Ltd for proof reading this paper.

\section{Funding Information}

This work was funded by the Vietnamese Ministry of Education and Curtin International Postgraduate Research Scholarship and conducted at Curtin University, Australia.

\section{Author's Contributions}

Ha Thi Thu Bui: As the correspondent author, as a part of the $\mathrm{PhD}$ thesis, who was responsible for setting up and running the experiment, collecting and analysing data, writing the manuscript.

Trong Quoc Luu: Helped with seaweeds collection, experimental setup and data collection.

Ravi Fotedar: Supervised the research, edit and approved the manuscript.

\section{Ethics}

This article is original material. All authors have read and approved the manuscript. No ethical issue that may arise after the publication of this manuscript.

\section{References}

Ahmad, S.H., M. Surif, W.M.W. Omar, M.N.B. Rosli and A.R.M. Nor, 2011. Nutrient uptake growth and chlorophyll content of green seaweed Ulva reticulata: Response to different source of inorganic nutrients. Empowering Science Technology and Innovation towards a Better Tomorrow, UMTAS.

Allan, G.L., B. Banens and D.S. Fielder, 2001. Developing commercial inland saline aquaculture in Australia: Part 2 Resource inventory and assessment. FRDC Project No. 98/335. Canberra Fisheries Research Development Corporation: Fisheries Research Development Corporation.
Allan, G.L. and D.S. Fielder, 1997. Inland saline aquaculture activities in New South Wales. Proceedings of the Inland Saline Aquaculture Workshop, Perth, Australian Centre for International Agricultural Research, pp: 14-15.

Ara, J., S. Ehteshamul-Haque, V. Sultana, A. Ghaffar and R. Qasim, 1997. Use of Sargassum species for the control of Meloidogyne javanica in Okra. Nematol. Mediterranea.

Atkinson, M.J. and S.V. Smith, 1983. C:N:P ratios of benthic marine plants. Limnol. Oceanography, 28: 568-574. DOI: 10.4319/lo.1983.28.3.0568

Balode, M., I. Purina, C. Beéchemin and S.Y. Maestrini, 1998. Effects of nutrient enrichment on the growth rates and community structure of summer phytoplankton from the Gulf of Riga, Baltic Sea. J. Plankton Res., 20: 2251-2272.

DOI: $10.1093 /$ plankt/20.12.2251

Bast, F. and C.S. Khem, 2014. An illustrated review on cultivation and life history of agronomically important seaplants. In: Seaweed: Mineral Composition, Nutritional and Antioxidant Benefits and Agricultural Uses, Nova Publishers, New York, pp: 39-70.

Blumwald, E., G.S. Aharon and M.P. Apse, 2000. Sodium transport in plant cells. Biochim. Biophys. Acta, 1465: 140-151.

DOI: 10.1016/S0005-2736(00)00135-8

Borowitzka, M.A., 1997. Algae. In: Inland Saline Aquaculture, Smith, B. and C. Barlow (Eds.), ACIAR, pp: 35-36. The Australian Centre for International Agricultural Research, ACIAR Proceeding No. 83. Perth.

Boyd, C.E. and T. Thunjai, 2003. Concentrations of major ions in waters of inland shrimp farms in china, Ecuador, Thailand and the united states. J. World Aquaculture Society, 34: 524-532. DOI: 10.1111/j.1749-7345.2003.tb00092.x

Bui, H.T.T. T.Q. Luu and R. Fotedar, 2017a. Growth feasibility of Lomentaria sp. in inland saline water. Environ. Risk Assess. Remediat., 1: 47-55

Bui, H.T.T., T.Q. Luu, R. Fotedar and U. Tantulo, 2017 b. Productivity of Sargassum linearifolium in potassium fortified inland saline water under laboratory conditions. Aquaculture Res., 48: 5631-5639. DOI: 10.1111/are.13385

Campbell, S., 2001. Ammonium requirements of fastgrowing ephemeral macroalgae in a nutrientenriched marine embayment (Port Phillip Bay, Australia). Inter-Res. Marine Ecol. Progress Series, 209: 99-107. DOI: 10.3354/meps209099

Chen, B. and D. Zou, 2014. Growth and photosynthetic activity of Sargassum henslowianum (Fucales, Phaeophyta) seedlings in responses to different light intensities, temperatures and $\mathrm{CO}_{2}$ levels under laboratory conditions. Marine Biol. Res., 10: 1019-1026. DOI: 10.1080/17451000.2013.872798 
Choi, H.G., K.H. Lee, H.I. Yoo, P.J. Kang and Y.S. Kim, 2009. Physiological differences in the growth of Sargassum horneri between the germling and adult stages. Proceedings of the 19th International Seaweed Symposium, (ISS' 09), pp: 279-285. DOI: 10.1007/978-1-4020-9619-8 35

Coutinho, R. and R. Zingmark, 1993. Interactions of light and nitrogen on photosynthesis and growth of the marine macroalga Ulva curvata (Kützing) De Toni. J. Exp. Marine Biol. Ecol., 167: 11-19.

DOI: 10.1016/0022-0981(93)90180-V

Diaz-Pulido, G. and L.J. Mccook, 2005. Effects of nutrient enhancement on the fecundity of a coral reef macroalga. J. Exp. Marine Biol. Ecol., 317: 13-24. DOI: $10.1016 /$ j.jembe.2004.11.013

Dinh, H.Q., 2016. Cultural biology of the blue mussel Mytilus Edulis (Linnaeus 1758) in inland saline water in Western Australia. PhD Thesis, Curtin University.

Doroudi, M.S., D.S. Fielder, G.L. Allan and G.K. Webster, 2006. Combined effects of salinity and potassium concentration on juvenile mulloway (Argyrosomus japonicus, Temminck and Schlegel) in inland saline groundwater. Aquaculture Res., 37: 1034-1039. DOI: $10.1111 /$ j.1365-2109.2006.01525.x

Downing, J.A., 1997. Marine nitrogen: Phosphorus stoichiometry and the global N:P cycle. Biogeochemistry, 37: 237-252. DOI: $10.1023 / \mathrm{A}: 1005712322036$

Evans, H.J. and G.J. Sorger, 1966. Role of mineral elements with emphasis on the univalent cations. Annual Rev. Plant Physiol., 17: 47-76. DOI: 10.1146/annurev.pp.17.060166.000403

Fielder, D.S. and G.L. Allan, 2003. Improving fingerling production and evaluating inland saline water culture of Snapper Pagrus auratus. NSW Fisheries Final Report Series No. 43. Nelson Bay: CRC Project No. C4.2

Fielder, D.S., W.J. Bardsley and G.L. Allan, 2001. Survival and growth of Australian snapper, Pagrus auratus, in saline groundwater from inland New South Wales, Australia. Aquaculture, 201: 73-90. DOI: 10.1016/S0044-8486(01)00555-5

Forsberg, J.A., P.W. Dorsett and W.H. Neill, 1996. Survival and growth of Red Drum Sciaenops ocellatus in saline groundwaters of West Texas, USA. J. World Aquaculture Society, 27: 462-474. DOI: $10.1111 /$ j.1749-7345.1996.tb00631.x

Fotedar, R., G. Krishna, U. Tantulo, I. Mcgregor and B. Phillips, 2011. Recent Developments. In: Recent Advances and New Species in Aquaculture, Fotedar, R. and B. Philips (Eds.), Blackwell Publishing Ltd., West Sussex, pp: 1-21.

Gao, K. and W. Hua, 1997. In situ growth rates of Sargassum horneri (Fucales Phaeophyta). Phycol. Res., 45: 55-57.

DOI: 10.1111/j.1440-1835.1997.tb00062.x
Hanisak, M.D. and M. Samuel, 1987. Growth rates in culture of several species of Sargassum from Florida, USA. Hydrobiologia, 151: 399-404. DOI: $10.1007 /$ BF00046159

Helrich, K.C., 1990. Official methods of Analysis of the AOAC. Virginia, USA Association of Official Analytical Chemists Inc. Arlington.

Huisman, J.M., 2000. Marine plants of Australia. Western Australia University of Western Australia Press.

Hur, S., H. Lee, Y. Kim, B.H. Lee and J. Shin, 2008. Sargaquinoic acid and sargachromenol, extracts of Sargassum sagamianum, induce apoptosis in Hacat cells and mice skin: Its potentiation of UVB-induced apoptosis. Eur. J. Pharmacol., 582: 1-11. DOI: $10.1016 /$ j.ejphar.2007.12.025

Hwang, R.L., C.C. Tsai and T.M. Lee, 2004. Assessment of temperature and nutrient limitation on seasonal dynamics among species of Sargassum from a coral reef in southern Taiwain. J. Phycol., 40: 463-473. DOI: 10.1111/j.1529-8817.2004.03086.x

Jie, B., T. Xiang-Li, D. Shuang-Lin and J. Hong-Bo, 2008. Effect of temperature salinity and light intensity on nitrogen and phosphorus uptake by Sargassum thunbergii. J. Fishery Sci. China.

Kirst, G.O., 1977. Ion composition of unicellular marine and fresh-water algae with special reference to Platymonas subcordiformis cultivated in media with different osmotic strengths. Oecologia, 28 177-189. DOI: $10.1007 / \mathrm{bf} 00345253$

Lapointe, B.E., 1986. Phosphorus-limited photosynthesis and growth of Sargassum natans and Sargassum fluitans (Phaeophyceae) in the western North Atlantic. Deep Sea Res., 33: 391-399. DOI: 10.1016/0198-0149(86)90099-3

Larned, S.T., 1998. Nitrogen- versus phosphorus-limited growth and sources of nutrients for coral reef macroalgae. Marine Biol., 132: 409-421. DOI: $10.1007 / \mathrm{s} 002270050407$

Lignell, A. and M. Pedersén, 1989. Effects of $\mathrm{pH}$ and inorganic carbon concentration on growth of Gracilaria secundata. British Phycol. J., 24: 83-89. DOI: 10.1080/00071618900650071

Liu, D., P. Amy and J. Sun, 2004. Preliminary study on the responses of three marine algae, Ulva pertusa (Chlorophyta), Gelidium amansii (Rhodophyta) and Sargassum enerve (Phaeophyta), to nitrogen source and its availability. J. Ocean Univ. China, 3: 75-79. DOI: $10.1007 / \mathrm{s} 11802-004-0013-\mathrm{z}$

Malhotra, B. and A.D.M. Glass, 1995. Potassium fluxes in Chlamydomonas reinhardtii (I.Kinetics and electrical potentials). Plant Physiol., 108: 1527-1536. PMID: 12228559

Martin-Smith, K.M., 1993. The phenology of four species of Sargassum at magnetic island Australia. Botanica Marina, 36: 327-334.

DOI: $10.1515 /$ botm.1993.36.4.327 
Martin-Smith, K.M., 1994. The role of epifaunal crustaceans on Sargassum spp. at Magnetic island Great Barriers Reef Australia. PhD Thesis, James Cook.

May-Lin, B.Y. and W. Ching-Lee, 2013. Seasonal growth rate of Sargassum species at Teluk Kemang, Port Dickson, Malaysia. J. Applied Phycol., 25: 805-814. DOI: 10.1007/s10811-012-9963-5

Mccourt, R.M., 1984. Seasonal patterns of abundance, distributions and phenology in relation to growth strategies of three Sargassum species. J. Exp. Marine Biol. Ecol., 74: 141-156.

DOI: 10.1016/0022-0981(84)90082-0

Mcdermid, K.J. and B. Stuercke, 2003. Nutritional composition of edible Hawaiian seaweeds. J. Applied Phycol., 15: 513-524.

DOI: 10.1023/B:JAPH.0000004345.31686.7f

Mourad, N., S. Kreydiyyeh J. Ghanawi and I.P. Saoud, 2012. Aquaculture of marine fish in inland low salinity well water: Potassium is not the only limiting element. Fisheries Aquaculture J., 42: 1-12. DOI: $10.4172 / 2150-3508.1000042$

Natarajan, S., K.P. Shanmugiahthevar and P.D. Kasi, 2009. Cholinesterase inhibitors from Sargassum and Gracilaria gracilis: Seaweeds inhabiting South Indian coastal areas (Hare Island, Gulf of Mannar). Natural Product Res., 23: 355-369. DOI: $10.1080 / 14786410802156036$

Ogburn, D.M., 1997. Environmental Considerations in the Use and Management of Inland Saline Water Bodies for Aquaculture. In: Smith B. and C. Barlow (Eds.), Inland Saline Aquaculture Perth, ACIAR, The Australian Centre for International Agricultural Research, ACIAR proceedings No. 83, pp: 32-34. Perth.

Pangestuti, R. and S.K. Kim, 2010. Neuroprotective properties of chitosan and its derivatives. Marine Drugs, 8: 2117-2128. DOI:10.3390/md8072117

Pérez-Mayorga, D.M., L.B. Ladah, J.A. ZertucheGonzález, J.J. Leichter and A.E. Filonov, 2011. Nitrogen uptake and growth by the opportunistic macroalga Ulva lactuca (linnaeus) during the internal tide. J. Exp. Marine Biol. Ecol., 406: 108-115. DOI: $10.1016 /$ j.jembe.2011.05.028

Perini, V. and M.E.S. Bracken, 2014. Nitrogen availability limits phosphorus uptake in an intertidal macroalga. Oecologia, 175: 667-676.

DOI: $10.1007 / \mathrm{s} 00442-014-2914-x$

Prangnell, D.I. and R. Fotedar, 2006a. Effect of sudden salinity change on Penaeus latisulcatus Kishinouye osmoregulation, ionoregulation and condition in inland saline water and potassium-fortified inland saline water. Comparative Biochem. Physiol., 145: 449-457. DOI: 10.1016/j.cbpa.2006.08.029
Prangnell, D.I. and R. Fotedar, 2006b. The growth and survival of western king prawns, Penaeus latisulcatus Kishinouye, in potassium-fortified inland saline water. Aquaculture, 259: 234-242. DOI: $10.1016 / \mathrm{j}$.aquaculture.2006.05.023

Reef, R., J.M. Pandolfi and C.E. Lovelock, 2012. The effect of nutrient enrichment on the growth, nucleic acid concentrations and elemental stoichiometry of coral reef macroalgae. Ecol. Evolut., 2: 1985-1995. DOI: $10.1002 /$ ece 3.330

Robards, K., I.D. Mckelvie, R.L. Benson, P.J. Worsfold, N.J. and Blundell, 1994. Determination of carbon, phosphorus, nitrogen and silicon species in waters. Analytica Chim. Acta, 287: 147-190. DOI: 10.1016/0003-2670(93)E0542-F

Schaffelke, B., 1999. Short-term nutrient pulses as tools to assess responses of coral reef macroalgae to enhanced nutrient availability. Marine Ecol. Progress Series, 182: 305-310. DOI: 10.3354/meps 182305

Schaffelke, B. and D.W. Klumpp, 1998. Nutrient-limited growth of the coral reef macroalga Sargassum baccularia and experimental growth enhancement by nutrient addition in continuous flow culture. Marine Ecol. Progress Series, 164: 199-211. DOI: $10.3354 /$ meps 164199

Smith, S.V., W.J. Kimmerer and T.W. Walsh, 1986. Vertical flux and biogeochemical turnover regulate nutrient limitation of net organic production in the north pacific gyre. Limnol. Oceanography, 31: 161-166. DOI: 10.4319/lo.1986.31.1.0161

Talling, J.F., 2010. Potassium-a non-limiting nutrient in fresh waters? Freshwater Rev., 3: 97-104.

DOI: $10.1608 /$ FRJ-3.2.1

Tantulo, U. and R. Fotedar, 2006. Comparison of growth, osmoregulatory capacity, ionic regulation and organosomatic indices of black tiger prawn (Penaeus monodon Fabricius, 1798) juveniles reared in potassium fortified inland saline water and ocean water at different salinities. Aquaculture, 258: 594605. DOI: 10.1016/j.aquaculture.2006.04.038

Thuy, T.T.T., B.M. Ly, T.T.T. Van, N.V. Quang, and H.C. Tu, 2015. Anti-HIV activity of fucoidans from three brown seaweed species. Carbohydrate Polymers, 115: 122-128.

DOI: $10.1016 / \mathrm{j}$. carbpol.2014.08.068

Tucker, C.S. and L.R. D'abramo, 2008. Managing high $\mathrm{pH}$ in freshwater ponds. Southern Regional Aquaculture Centre United States Department of Agriculture Cooperative State Research Education and Extension Service

Vuki, V.C. and I.R. Price, 1994. Seasonal changes in the Sargassum populations on a fringing coral reef, magnetic island, great barrier reef region, Australia. Aquatic Botany, 48: 153-166.

DOI: 10.1016/0304-3770(94)90082-5 
Wallentinus, I., 1984. Comparisons of nutrient uptake rates for Baltic macroalgae with different thallus morphologies. Marine Biol., 80: 215-225. DOI: $10.1007 / \mathrm{BF} 02180189$

Womersley, H.B.S., 1987. The marine benthic flora of southern Australia. Part II Adelaide South Australian Government Printing Devision.

Womersley, H.B.S., 1996. The marine benthic flora of southern Australia: Rhodophyta Part IIIB. Gracilarialse Rhodymeniales Corallinales and Bonnemaisoniales Canberra and Adelaide Australian Biological Resources Study and the State Herbarium of South Australia.
Yamauchi, K., 1984. The formation of Sargassum beds on artificial substrata by transplanting seedlings of S. horneri (Turner) C. Agardh and S. muticum (Yendo) fensholt. Nippon Suisan Gakkaishi, 50: 1115-1123. DOI: 10.2331/suisan.50.1115

Yende, S.R., U.N. Harle and B.B. Chaugule, 2014. Therapeutic potential and health benefits of Sargassum species. Pharmacognosy Rev., 8: 1-7. DOI: $10.4103 / 0973-7847.125514$ 